Article

\title{
Syntheses and Structure-Activity Relationships of $N$-Phenethyl-Quinazolin-4-yl-Amines as Potent Inhibitors of Cytochrome bd Oxidase in Mycobacterium tuberculosis
}

\author{
Sarah M. Hopfner ${ }^{1}{ }^{\mathbb{D}}$, Bei Shi Lee ${ }^{2}$, Nitin P. Kalia ${ }^{3} \mathbb{D}$, Marvin J. Miller ${ }^{4}$, Kevin Pethe ${ }^{2,5}$ \\ and Garrett C. Moraski ${ }^{1, *(\mathbb{D})}$
}

1 Department of Chemistry and Biochemistry, Montana State University, 103 Chemistry and Biochemistry Building, Bozeman, Montana, MT 59717, USA; sarah.hopfner@student.montana.edu

2 School of Biological Sciences, Nanyang Technological University, Singapore 637551, Singapore; beishi001@e.ntu.edu.sg (B.S.L.); kevin.pethe@ntu.edu.sg (K.P.)

3 Department of Biological Sciences, National Institute of Pharmaceutical Education and Research, Hyderabad, Telangana 500037, India; kalianpk@gmail.com

4 Department of Chemistry and Biochemistry, University of Notre Dame, 251 Nieuwland Science Hall, Notre Dame, Indiana, IN 46556, USA; mmiller1@nd.edu

5 Lee Kong Chian School of Medicine, Nanyang Technological University, Experimental Medicine Building, 59 Nanyang Drive, Singapore 636921, Singapore

* Correspondence: garrett.moraski@montana.edu

check for updates

Citation: Hopfner, S.M.; Lee, B.S.; Kalia, N.P.; Miller, M.J.; Pethe, K.; Moraski, G.C. Syntheses and Structure-Activity Relationships of N-Phenethyl-Quinazolin-4-yl-Amines as Potent Inhibitors of Cytochrome bd Oxidase in Mycobacterium tuberculosis. Appl. Sci. 2021, 11, 9092. https:// doi.org/10.3390/app11199092

Academic Editor: Alfonso Zambon

Received: 10 August 2021

Accepted: 22 September 2021

Published: 29 September 2021

Publisher's Note: MDPI stays neutral with regard to jurisdictional claims in published maps and institutional affiliations.

Copyright: (c) 2021 by the authors. Licensee MDPI, Basel, Switzerland. This article is an open access article distributed under the terms and conditions of the Creative Commons Attribution (CC BY) license (https:// creativecommons.org/licenses/by/ $4.0 /)$.

\begin{abstract}
The development of cytochrome $b d$ oxidase (cyt- $b d$ ) inhibitors are needed for comprehensive termination of energy production in Mycobacterium tuberculosis $(\mathrm{Mtb})$ to treat tuberculosis infections. Herein, we report on the structure-activity-relationships (SAR) of 22 new N-phenethylquinazolin-4-yl-amines that target cyt- $b d$. Our focused set of compounds was synthesized and screened against three mycobacterial strains: Mycobacterium bovis BCG, Mycobacterium tuberculosis H37Rv and the clinical isolate Mycobacterium tuberculosis N0145 with and without the cytochrome bcc: $a a_{3}$ inhibitor Q203 in an ATP depletion assay. Two compounds, 12a and 19a, were more active against all three strains than the naturally derived cyt- $b d$ inhibitor aurachin D.
\end{abstract}

Keywords: tuberculosis; drug development; structure-activity relationships; quinazoline; energy metabolism; cytochrome $b d$ oxidase

\section{Introduction}

Tuberculosis (TB) is still a leading cause of death from an infectious agent. According to the World Health Organization's 2020 Global Tuberculosis Report, over 10 million people became ill with the disease in 2019 [1]. Action must be taken to combat this continuing public health threat. TB is caused by the bacterium Mycobacterium tuberculosis (Mtb) and spread by aerosols of an infected person through a cough or sneeze. Treatment of individuals infected with TB consists of several medications that are taken together for 6-9 months. First-line drugs include isoniazid (INH), rifampin (RIF), ethambutol (EMB) and pyrazinamide (PZA) [1]. Other barriers, such as lengthy treatment regimens and access to care, have made adherence to treatment challenging. Shorter treatment schedules are strongly desired. Despite the advances in drug therapies and modern medications, such as bedaquiline, delamanid and pretomanid, there is a need for comprehensive drug combinations and new treatment regimens to combat drug resistant TB [2]. Consideration of how Mtb survives onslaughts of chemotherapies gives insight into more effective treatment. Investigating the mechanism of action of antibiotics provides critical information that can be used to ultimately cure TB infections [3]. Targets within energy metabolism processes, specifically those involved in the oxidative phosphorylation pathway, have become of increased interest. Mtb can persist in a hypoxic, non-replicating, drug-tolerant 
state [4]. It has been demonstrated that interrupting oxidative phosphorylation in $\mathrm{Mtb}$ is an encouraging strategy [5-7]. While compounds have been made to inhibit various targets within the oxidative phosphorylation pathway, limitations have been observed, including the inability to inhibit oxygen respiration and clear Mtb infection due to a redundancy between the terminal oxidases cytochrome $b c c: a a_{3}$ (cyt-bcc:aa $a_{3}$ ) and cytochrome $b d$ (cyt- $b d$ ) [8]. Cytochrome $b c c: a a_{3}$ is the main aerobic terminal oxidase in Mtb [9]. The compound Q203 (4, Telacebec ${ }^{\circledR}$ ) inhibits cytochrome $b c c$ by binding to the QcrB subunit, thereby inhibiting the function of the cytochrome $b c c: a a_{3}$ super-complex [10]. When cytochrome $b c c: a a_{3}$ is inactive, $c y t-b d$ is upregulated [11]. This adaptation provides protection against cytochrome $b c c: a a_{3}$ inhibitors such as Q203. We have shown that targeting cyt-bd in combination with Q203 is bactericidal against replicating and non-replicating Mtb [12]. This makes cyt-bd an enticing target for the development of antituberculosis agents to effectively eliminate infection.

Interested in the inhibition of this target, we screened a small set (56) of antibacterial compounds that originated from our decades-old antibacterial program. This led to the identification of cyt- $b d$ compounds like the thieno[3,2- $d$ ]pyrimidin-4-amines in which (1) is an example, the in vivo active optimized inhibitor ND-11992 (2) and hit compound 3 (Figure 1) [13].<smiles>CC(C)(C)c1ccc(CCNc2ncnc3ccsc23)cc1</smiles>
1<smiles>CCc1nc2ccc(Cl)cn2c1C(=O)NCc1ccc(N2CCC(c3ccc(OC(F)(F)F)cc3)CC2)cc1</smiles>

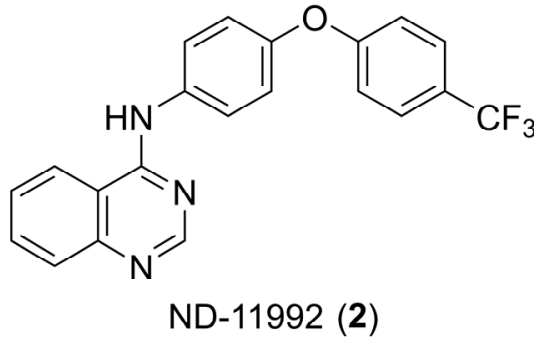

ND-11992 (2)<smiles>c1ccc(CCNc2ncnc3ccccc23)cc1</smiles><smiles>CC(C)=CCCC(C)=CCCC(C)=CCc1c(C)[nH]c2ccccc2c1=O</smiles>

Figure 1. Known synthetically accessible small molecule cyt- $b d$ inhibitors: thieno[3,2- $d$ ]pyrimidin-4-amine (1) [13], ND11992 (2) [12], and N-phenethylquinazolin-4-amine (3) screening hit. The selective cyt-bcc:aa 3 inhibitor Q203 (4) [10] and natural cyt-bd inhibitor aurachin D [7].

The $N$-phenethylquinazolin-4-amine class, exemplified by compound 3 (Figure 1), has an $\mathrm{IC}_{50}$ of $11 \mu \mathrm{M}$ against Mycobacterium bovis BCG and an $\mathrm{IC}_{50}$ of $27 \mu \mathrm{M}$ against Mycobacterium tuberculosis H37Rv as determined by our published ATP depletion assay [13]. This assay is purposefully run in the presence and absence of Q203 (4, a selective cyt$b c c: a a_{3}$ inhibitor) to indicate whether a compound inhibits alone or synergizes due to the conditional essentiality of cyt- $b d$ to maintain ATP homeostasis once cyt-bcc:aa 3 is selectively inhibited by Q203. Compound 3 was inactive $\left(\mathrm{IC}_{50}>25 \mu \mathrm{M}\right)$ in the absence of Q203 which is an indication of on-target potency based upon assay design. Similarly, compound 3 was amenable to chemical modifications and structure-activity relationship (SAR) development. Herein, we report our initial design, synthesis, and activity assessment of various quinazoline compounds for activity against cyt-bd of Mycobacterium bovis BCG and Mycobacterium tuberculosis. 


\section{Materials and Methods}

\subsection{Chemistry}

General. All anhydrous solvents, reagent grade solvents for chromatography and starting materials were purchased from either Aldrich Chemical Co. (Milwaukee, WI, USA) or Fisher Scientific (Suwanee, GA, USA) unless otherwise noted. Water was distilled and purified through a Milli-Q water system (Millipore Corp., Bedford, MA, USA). General methods of purification of compounds involved the use of silica cartridges purchased from Practichem, LLC. (www.practichem.com, last accessed on 23 September 2021) and/or recrystallization. The reactions were monitored by TLC on precoated Merck 60 F254 silica gel plates and visualized using UV light $(254 \mathrm{~nm})$. All compounds were analyzed for purity by HPLC and characterized by ${ }^{1} \mathrm{H}$ and ${ }^{13} \mathrm{C}$ NMR using a Bruker Ascend Avance III HD Spectrometer $(500 \mathrm{MHz})$. Chemical shifts are reported in ppm $(\delta)$ relative to the residual solvent peak in the corresponding spectra; chloroform $\delta 7.27$ and $\delta 77.23$, methanol $\delta 3.31$ and $\delta 49.00$ and coupling constants $(J)$ are reported in hertz $(\mathrm{Hz})$ (where, $\mathrm{s}=$ singlet, $\mathrm{bs}=$ broad singlet, $\mathrm{d}=$ doublet, $\mathrm{dd}=$ double doublet, $\mathrm{bd}=$ broad doublet, $\mathrm{ddd}=$ double doublet of doublet, $\mathrm{t}=$ triplet, $\mathrm{tt}=$ triple triplet, $\mathrm{q}=$ quartet, $\mathrm{m}=$ multiplet) and analyzed using MestreNova NMR data processing. ${ }^{19} \mathrm{~F}$ NMR were run without a standard and are uncorrected. Mass spectra values are reported as $\mathrm{m} / \mathrm{z}$. Melting points were measured on a Buchi B-545 melting point instrument and are uncorrected, measured against benzoic acid 118.8-120.2 ${ }^{\circ} \mathrm{C}$. Compounds 3, 6a-26a appear within US Provisional Patent Application No. $62 / 783,984$. The quinazolines $(5, \mathbf{1 6}-\mathbf{2 6})$ and amines $(\mathbf{6 - 1 5})$ used in synthesis are all commercially available.

LC-MS method: The liquid chromatography-mass spectrometry method was performed on an Agilent 1290 infinity coupled to Agilent 6538 Ultra High-Definition Quadrupole Time of Flight (UHD-QTOF) instrument. A separation was achieved by using reverse phase Waters Acuity UPLC HSS T3 $1.8 \mu \mathrm{m}(2.1 \times 100 \mathrm{~mm})$ column from Waters (Milford, MA, USA). All solvents were purchased from Fischer Scientific LCMS Optima grade solvents. Water containing $0.1 \%$ formic acid was used as mobile phase A and acetonitrile containing $0.1 \%$ formic acid was used as mobile phase B. The injection volume was set at $1 \mu \mathrm{L}$. Samples were injected in a gradient of $95 \%$ mobile phase $\mathrm{A}$ and $5 \%$ mobile phase $\mathrm{B}$ in the initial condition to $5 \%$ mobile phase $A$ and $95 \%$ mobile phase $B$ in 9 min. The eluent was held at that composition for an additional $3 \mathrm{~min}$ and switched back to the initial condition at $12 \mathrm{~min}$.

The MS data acquisition was performed from $50-1000 \mathrm{~m} / \mathrm{z}$ at 1.0 spectra/sec scan rate. The source gas temperature was set at $350{ }^{\circ} \mathrm{C}$ with a flow of $81 / \mathrm{min}$. The nebulizer gas was set at 55 psig. The capillary voltage was set at 3500 volts with fragmentor at 100 , skimmer at 45 and octopole RF 500 volts. Prior to sample runs, the instrument was calibrated using Agilent low mass calibrant solution.

Data analysis: The data collected in Agilent LC-MS was analyzed using Agilent Mass Hunter software for HRMS calculation.

\section{General Syntheses of N-Phenethylquinazolin-4-Amine Analogs}

General procedure A for base catalyzed $S_{N} A r$ reaction for the preparation of compounds $6 \mathbf{a}, 7 \mathrm{a}, 9 \mathrm{9a}, 12 \mathrm{a}-26 \mathrm{a}$. In a sealed vial, 4-chloroquinazoline $(5,100 \mathrm{mg}, 0.61 \mathrm{mmol}$, 1 equiv.), 2-[4-(trifluoromethoxy)phenyl]ethylamine (7, $125 \mathrm{mg}, 0.61 \mathrm{mmol}, 1$ equiv.) and potassium carbonate $(84 \mathrm{mg}, 0.61 \mathrm{mmol}, 1$ equiv. were combined in DMSO $(4 \mathrm{~mL})$. The reaction was heated to $100{ }^{\circ} \mathrm{C}$ for $12 \mathrm{~h}$. The reaction mixture was concentrated to dryness. The residue was extracted with $\mathrm{CH}_{2} \mathrm{Cl}_{2}$ and washed with $5 \%$ aqueous acetic acid solution (2x), water and brine. The organic phase was collected, dried over sodium sulfate, filtered, and concentrated in vacuo. Crude material obtained was purified by either silica gel chromatography with a gradient of $\mathrm{CH}_{2} \mathrm{Cl}_{2}$ : ethyl acetate: solvent system $(0-80 \%)$ or recrystallized from hot isopropanol or acetonitrile to afford the product.

General procedure B for acid catalyzed $\mathrm{S}_{\mathrm{N}} \mathrm{Ar}$ reaction for the preparation of compounds 3, 8a, 10a, 11a. In a sealed vial, desired 4-chloroquinazoline 16-26 (1 equiv.) and 2-[4-(trifluoromethoxy)phenyl]ethylamine (7, 1 equiv.) were dissolved in a 3:1 tetrahydrofu- 
ran: 2-propanol solution ( $4 \mathrm{~mL})$. Next, was added $12 \mathrm{M} \mathrm{HCl}(\sim 0.4$ equiv.). The solution was heated at $70^{\circ} \mathrm{C}$ for $24 \mathrm{~h}$. The reaction was concentrated to dryness. The residue was dissolved in $\mathrm{CH}_{2} \mathrm{Cl}_{2}$ and washed with saturated aqueous $\mathrm{NaHCO}_{3}$ solution, water, and brine. The organic phase was collected, dried over sodium sulfate, filtered, and concentrated in vacuo. Crude material obtained was purified by either silica gel column chromatography with a gradient of $\mathrm{CH}_{2} \mathrm{Cl}_{2}$ : ethyl acetate: solvent system $(0-80 \%)$ or recrystallized from hot isopropanol or acetonitrile to afford the product.

N-Phenethylquinazolin-4-amine, 3

Following general procedure B, using 4-chloroquinazoline (5, $100 \mathrm{mg}, 0.59 \mathrm{mmol})$, 2-phenylethylamine (72 $\mathrm{mg}, 0.59 \mathrm{mmol})$, and $12 \mathrm{~N} \mathrm{HCl}(11 \mu \mathrm{L}, 0.14 \mathrm{mmol})$ gave 3 as pale-yellow crystals $(43 \mathrm{mg}, 28 \%)$. mp $171.5-171.9{ }^{\circ} \mathrm{C} ;{ }^{1} \mathrm{H}(500 \mathrm{MHz}, \mathrm{MeOD}) \delta \mathrm{ppm} 8.47$ $(\mathrm{s}, 1 \mathrm{H}), 8.06(\mathrm{~d}, J=8.3 \mathrm{~Hz}, 1 \mathrm{H}), 7.82-7.77(\mathrm{~m}, 1 \mathrm{H}), 7.72(\mathrm{~d}, J=8.3 \mathrm{~Hz}, 1 \mathrm{H}), 7.55-7.50(\mathrm{~m}$, $1 \mathrm{H}), 7.31-7.26(\mathrm{~m}, 4 \mathrm{H}), 7.23-7.17(\mathrm{~m}, 1 \mathrm{H}), 3.86(\mathrm{t}, J=7.4 \mathrm{~Hz}, 2 \mathrm{H}), 3.04(\mathrm{t}, J=7.4 \mathrm{~Hz}, 2 \mathrm{H}){ }^{13} \mathrm{C}$ (125 MHz, MeOD) $\delta$ ppm 160.0, 154.7, 148.2, 139.3, 132.7, 128.5, 128.1, 126.3, 126.0, 125.9, $121.9,115.1,42.5,34.7$. HRMS (EI), $\mathrm{M}+1$ calculated for $\mathrm{C}_{16} \mathrm{H}_{15} \mathrm{~N}_{3}, 250.1339$, found 250.1351. These experimental results are consistent with previous reports [14-16].

Quinazolin-4-yl\{2-[4-(trifluoromethyl)phenyl]ethyl\}amine, 6a

Following general procedure A, using 4-chloroquinazoline (5, $100 \mathrm{mg}, 0.59 \mathrm{mmol})$, 2-[4-(trifluoromethyl)phenyl]ethylamine $(114 \mathrm{mg}, 0.59 \mathrm{mmol})$ and potassium carbonate (81 mg, $0.59 \mathrm{mmol})$ gave $6 \mathrm{a}$ as gold crystals $(79 \mathrm{mg}, 38 \%) . \mathrm{mp} 160.3-161.0{ }^{\circ} \mathrm{C} ;{ }^{1} \mathrm{H}(500 \mathrm{MHz}$, $\left.\mathrm{CDCl}_{3}\right) \delta$ ppm $8.73(\mathrm{~s}, 1 \mathrm{H}), 7.88(\mathrm{~d}, J=7.9 \mathrm{~Hz}, 1 \mathrm{H}), 7.77(\mathrm{ddd}, J=8.4,7.0,1.3 \mathrm{~Hz}), 7.63-7.57$ $(\mathrm{m}, 3 \mathrm{H}), 7.47$ (ddd, $J=8.2,7.0,1.2 \mathrm{~Hz}), 7.40$ (d, $J=8.0 \mathrm{~Hz}, 2 \mathrm{H}), 5.79$ (br.s, 1H), 3.99 (dt,

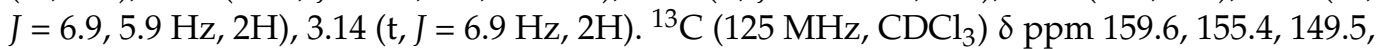
$143.2(\mathrm{~d}, J=1.5 \mathrm{~Hz}), 132.7,129.2,129.1(\mathrm{q}, J=32.4 \mathrm{~Hz}), 128.2,126.1,125.6(\mathrm{q}, J=3.8 \mathrm{~Hz})$, $124.7(\mathrm{q}, J=271.9 \mathrm{~Hz}), 120.2,114.9,42.1,35.2 .{ }^{19} \mathrm{~F}\left(470 \mathrm{MHz}, \mathrm{CDCl}_{3}\right) \delta \mathrm{ppm}-62.4(\mathrm{~s}, 3 \mathrm{~F})$. HRMS (EI), $\mathrm{M}+1$ calculated for $\mathrm{C}_{17} \mathrm{H}_{14} \mathrm{~F}_{3} \mathrm{~N}_{3}, 318.1213$, found 318.1229. This compound appears in referenced patent and fungicidal activity was reported [17].

Quinazolin-4-yl\{2-[4-(trifluoromethoxy)phenyl]ethyl\}amine, 7a

Following general procedure A, using 4-chloroquinazoline (5, $100 \mathrm{mg}, 0.59 \mathrm{mmol})$, 2-[4-(trifluoromethoxy)phenyl]ethylamine $(125 \mathrm{mg}, 0.59 \mathrm{mmol})$ and potassium carbonate $(84 \mathrm{mg}, 0.59 \mathrm{mmol})$ gave $7 \mathrm{a}$ as white crystals $(85 \mathrm{mg}, 42 \%) . \mathrm{mp} 137.4-137.8{ }^{\circ} \mathrm{C} ;{ }^{1} \mathrm{H}(500 \mathrm{MHz}$, $\left.\mathrm{CDCl}_{3}\right) \delta \mathrm{ppm} 8.73(\mathrm{~s}, 1 \mathrm{H}), 7.88(\mathrm{~d}, J=8.2 \mathrm{~Hz}, 1 \mathrm{H}), 7.77(\mathrm{ddd}, J=8.4,7.0,1.3 \mathrm{~Hz}, 1 \mathrm{H})$, $7.60-7.56(\mathrm{~m}, 1 \mathrm{H}), 7.48(\mathrm{ddd}, J=8.3,7.0,1.1 \mathrm{~Hz}), 7.33-7.26(\mathrm{~m}, 2 \mathrm{H}), 7.20(\mathrm{~d}, J=8.0 \mathrm{~Hz}, 2 \mathrm{H})$, 5.73 (br.s, $1 \mathrm{H}), 3.97(\mathrm{dt}, J=7.0,5.9 \mathrm{~Hz}, 2 \mathrm{H}), 3.08(\mathrm{t}, J=7.0 \mathrm{~Hz}, 2 \mathrm{H}) .{ }^{13} \mathrm{C}\left(125 \mathrm{MHz}, \mathrm{CDCl}_{3}\right) \delta$ ppm 159.3, 155.4, 149.5, $148.0(\mathrm{~d}, J=1.8 \mathrm{~Hz}), 137.7,132.6,130.2,128.8,126.1,121.3,120.6(\mathrm{q}$, $J=235.0 \mathrm{~Hz}), 120.1,114.9,42.3,34.6 .{ }^{19} \mathrm{~F}\left(470 \mathrm{MHz}, \mathrm{CDCl}_{3}\right) \delta \mathrm{ppm}-59.9(\mathrm{~s}, 3 \mathrm{~F}) . \mathrm{HRMS}$ (EI), $\mathrm{M}+1$ calculated for $\mathrm{C}_{17} \mathrm{H}_{14} \mathrm{~F}_{3} \mathrm{~N}_{3} \mathrm{O}, 334.1162$, found 334.1155 .

[2-(4-Chlorophenyl)ethyl]quinazolin-4-ylamine, 8a

Following general procedure B, using 4-chloroquinazoline (5, $100 \mathrm{mg}, 0.59 \mathrm{mmol})$, 2-(4-chlorophenyl)ethylamine (94 mg, $0.59 \mathrm{mmol})$ and $12 \mathrm{~N} \mathrm{HCl} \mathrm{(11} \mu \mathrm{L}, 0.14 \mathrm{mmol})$ gave 8a as yellow crystals (35 mg, 20\%). mp 191.0-192.1 ${ }^{\circ} \mathrm{C} ;{ }^{1} \mathrm{H}(500 \mathrm{MHz}, \mathrm{MeOD}) \delta \mathrm{ppm} 8.47$ $(\mathrm{s}, 1 \mathrm{H}), 8.05(\mathrm{dd}, J=8.3,0.7 \mathrm{~Hz}, 1 \mathrm{H}), 7.80(\mathrm{ddd}, J=8.3,7.0,1.3 \mathrm{~Hz}, 1 \mathrm{H}), 7.73-7.70(\mathrm{~m}, 1 \mathrm{H})$, $7.53(\mathrm{ddd}, J=8.3,7.0,1.3 \mathrm{~Hz}, 1 \mathrm{H}), 7.30-7.25(\mathrm{~m}, 2 \mathrm{H}), 3.88-3.83(\mathrm{~m}, 2 \mathrm{H}), 3.03(\mathrm{t}, J=7.3 \mathrm{~Hz}$, 2H). ${ }^{13} \mathrm{C}(125 \mathrm{MHz}, \mathrm{MeOD}) \delta$ ppm 160.0, 154.6, 148.2, 138.1, 132.8, 131.8, 130.2, 128.1, 126.3, $126.1,121.9,115.1,42.2,34.0$. HRMS (EI), $\mathrm{M}+1$ calculated for $\mathrm{C}_{16} \mathrm{H}_{14} \mathrm{ClN}_{3}, 284.0949$, found 284.0950. These experimental results are consistent with previous reports [18-20].

[2-(4-(Pentafluoro- $(\lambda)^{6}$-sulfaneyl]quinazolin-4-ylamine, 9a

Following general procedure A, using 4-chloroquinazoline $(5,70 \mathrm{mg}, 0.42 \mathrm{mmol})$ and 2-(4-(pentafluoro- $(\lambda)^{6}$-sulfaneyl)phenyl)ethan-1-amine $(105 \mathrm{mg}, 0.42 \mathrm{mmol})$ and potassium carbonate (59 mg, $0.42 \mathrm{mmol})$ gave $9 \mathrm{a}$ as white crystals $(97 \mathrm{mg}, 61 \%)$. mp $169.2-170.7^{\circ} \mathrm{C}$; 
${ }^{1} \mathrm{H}\left(500 \mathrm{MHz}, \mathrm{CDCl}_{3}\right) \delta \mathrm{ppm} 8.73(\mathrm{~s}, 1 \mathrm{H}), 7.89(\mathrm{~d}, J=8.3 \mathrm{~Hz}, 1 \mathrm{H}), 7.80-7.70(\mathrm{~m}, 3 \mathrm{H}), 7.61(\mathrm{~d}$, $J=8.2 \mathrm{~Hz}, 1 \mathrm{H}), 7.51-7.46(\mathrm{~m}, 1 \mathrm{H}), 7.37(\mathrm{~d}, J=8.3 \mathrm{~Hz}, 2 \mathrm{H}), 5.80$ (br.s, $1 \mathrm{H}), 3.99(\mathrm{q}, J=6.6 \mathrm{~Hz}$,

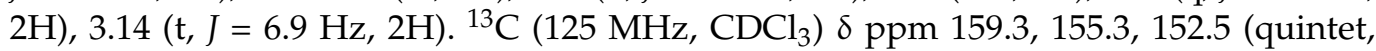
$J=17.6 \mathrm{~Hz}$ ), 149.5, 143.2, 132.7, 129.1, 128.8, 126.3 (quintet, $J=4.5 \mathrm{~Hz}$ ), 120.2, 114.9, 42.1, 34.9. ${ }^{19} \mathrm{~F}\left(470 \mathrm{MHz}, \mathrm{CDCl}_{3}\right) \delta \mathrm{ppm} 84.8$ (pent, $\left.J=150.0 \mathrm{~Hz}, 1 \mathrm{~F}\right), 63.1(\mathrm{~d}, J=150.0 \mathrm{~Hz}, 4 \mathrm{~F})$. HRMS (EI), $\mathrm{M}+1$ calculated for $\mathrm{C}_{16} \mathrm{H}_{14} \mathrm{~F}_{5} \mathrm{~N}_{3} \mathrm{~S}$, 376.0901, found 376.0908.

[2-(4-Methylpheny)ethyl]quinazolin-4-ylamine, 10a

Following general procedure B, using 4-chloroquinazoline $(5,100 \mathrm{mg}, 0.59 \mathrm{mmol})$, 2-(4-methylphenyl)ethan-1-amine ( $82 \mathrm{mg}, 0.59 \mathrm{mmol})$ and $12 \mathrm{~N} \mathrm{HCl}(11 \mu \mathrm{L}, 0.14 \mathrm{mmol})$ gave 10a as white crystals $(25 \mathrm{mg}, 15 \%) . \mathrm{mp} 183.6-184.6{ }^{\circ} \mathrm{C} ;{ }^{1} \mathrm{H}\left(500 \mathrm{MHz}, \mathrm{CDCl}_{3}\right) \delta \mathrm{ppm}$ $8.71(\mathrm{~s}, 1 \mathrm{H}), 7.86(\mathrm{~d}, J=8.2 \mathrm{~Hz}, 1 \mathrm{H}), 7.74(\mathrm{ddd}, J=8.3,7.0,1.3 \mathrm{~Hz}, 1 \mathrm{H}), 7.56(\mathrm{~d}, J=7.8 \mathrm{~Hz}$, $1 \mathrm{H}), 7.44(\mathrm{ddd}, J=8.2,7.0,1.1 \mathrm{~Hz}, 1 \mathrm{H}), 5.79$ (br.s, $1 \mathrm{H}), 3.95(\mathrm{dt}, J=6.7,5.8 \mathrm{~Hz}, 2 \mathrm{H}), 3.02(\mathrm{t}$,

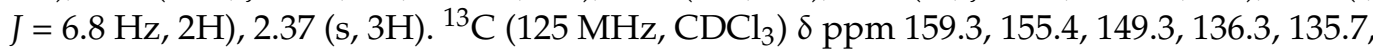
$132.6,129.5,128.7,128.6,126.0,120.3,115.0,42.3,34.8,21.1$. HRMS (EI), M + 1 calculated for $\mathrm{C}_{17} \mathrm{H}_{17} \mathrm{~N}_{3}, 264.1495$, found 264.1496.

\section{[2-(4-Methoxyphenyl)ethyl]quinazolin-4-ylamine, 11a}

Following general procedure B, using 4-chloroquinazoline $(5,100 \mathrm{mg}, 0.59 \mathrm{mmol})$, 2-(4-methoxyphenyl)ethylamine $(91 \mathrm{mg}, 0.59 \mathrm{mmol})$ and $12 \mathrm{~N} \mathrm{HCl}(11 \mu \mathrm{L}, 0.14 \mathrm{mmol})$ gave 11a as off-white crystals $(74 \mathrm{mg}, 43 \%)$. mp $172.3-172.9^{\circ} \mathrm{C} ;{ }^{1} \mathrm{H}\left(500 \mathrm{MHz}, \mathrm{CDCl}_{3}\right) \delta \mathrm{ppm} 8.71$ $(\mathrm{s}, 1 \mathrm{H}), 7.86(\mathrm{~d}, J=8.3 \mathrm{~Hz}, 1 \mathrm{H}), 7.74(\mathrm{ddd}, J=8.3,7.0,1.3 \mathrm{~Hz}, 1 \mathrm{H}), 7.56(\mathrm{~d}, J=8.3 \mathrm{~Hz}, 1 \mathrm{H})$, $7.45(\mathrm{ddd}, J=8.2,7.0,1.2 \mathrm{~Hz}, 1 \mathrm{H}), 7.20(\mathrm{dt}, J=8.6,2.9 \mathrm{~Hz}, 2 \mathrm{H}), 6.90(\mathrm{dt}, J=8.6,2.9 \mathrm{~Hz}, 2 \mathrm{H})$, $5.78(\mathrm{~s}, 1 \mathrm{H}), 3.93(\mathrm{dt}, J=6.8,5.7 \mathrm{~Hz}, 2 \mathrm{H}), 3.83(\mathrm{~s}, 3 \mathrm{H}), 3.01(\mathrm{t}, J=6.8 \mathrm{~Hz}, 2 \mathrm{H}) .{ }^{13} \mathrm{C}(125 \mathrm{MHz}$, $\left.\mathrm{CDCl}_{3}\right) \delta \mathrm{ppm} 159.3,158.4,155.4,149.4,132.6,130.8,129.8,128.6,126.0,120.3,115.0,114.2$, 55.3, 42.4, 34.3. HRMS (EI), $\mathrm{M}+1$ calculated for $\mathrm{C}_{17} \mathrm{H}_{17} \mathrm{~N}_{3} \mathrm{O}, 280.1444$, found 280.1458.

\section{\{2-[4-(tert-Butyl)phenyl]ethyl\}quinazolin-4-ylamine, 12a}

Following general procedure A, using 4-chloroquinazoline $(5,100 \mathrm{mg}, 0.59 \mathrm{mmol})$, 2-[4-(tert-butyl)phenyl]ethylamine $(110 \mathrm{mg}, 0.59 \mathrm{mmol})$ and potassium carbonate $(81 \mathrm{mg}$, $0.59 \mathrm{mmol}$ ) gave 12a as yellow crystals $(58 \mathrm{mg}, 29 \%)$ mp $149.5-151.3{ }^{\circ} \mathrm{C} ;{ }^{1} \mathrm{H}(500 \mathrm{MHz}$, $\left.\mathrm{CDCl}_{3}\right) \delta \mathrm{ppm} 8.71(\mathrm{~s}, 1 \mathrm{H}), 7.86(\mathrm{dd}, J=8.4,0.6 \mathrm{~Hz}, 1 \mathrm{H}), 7.74(\mathrm{ddd}, J=8.4,7.0,1.3 \mathrm{~Hz}, 1 \mathrm{H})$, $7.57(\mathrm{dd}, J=8.3,0.8 \mathrm{~Hz}, 1 \mathrm{H}), 7.45(\mathrm{ddd}, J=8.2,7.0,1.2 \mathrm{~Hz}, 1 \mathrm{H}), 7.39(\mathrm{dt}, J=8.3,2.3 \mathrm{~Hz}, 2 \mathrm{H})$, $7.22(\mathrm{dt}, J=8.3,2.2 \mathrm{~Hz}, 2 \mathrm{H}), 5.81$ (br.s, $1 \mathrm{H}), 3.96(\mathrm{dt}, J=6.8,5.7 \mathrm{~Hz}, 2 \mathrm{H}), 3.04(\mathrm{t}, J=6.8 \mathrm{~Hz}$,

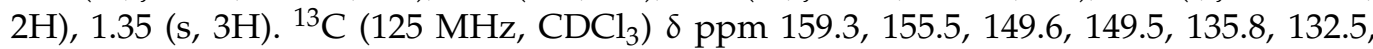
128.7, 128.5, 125.9, 125.7, 120.3, 115.0, 42.3, 34.7, 34.5, 31.4. HRMS (EI), M + 1 calculated for $\mathrm{C}_{20} \mathrm{H}_{23} \mathrm{~N}_{3}, 306.1965$, found 306.1967 . These experimental results are consistent with a previous report [21].

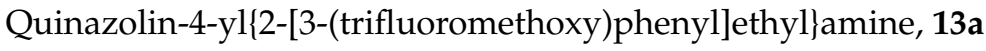

Following general procedure A, using 4-chloroquinazoline $(5,100 \mathrm{mg}, 0.59 \mathrm{mmol})$, 3-(trifluoromethoxy)phenylamine ( $125 \mathrm{mg}, 0.59 \mathrm{mmol})$ and potassium carbonate $(84 \mathrm{mg}$, $0.59 \mathrm{mmol})$ gave $13 \mathrm{a}$ as white crystals $(136 \mathrm{mg}, 67 \%)$ mp $144.0-144.9{ }^{\circ} \mathrm{C} ;{ }^{1} \mathrm{H}(500 \mathrm{MHz}$, $\left.\mathrm{CDCl}_{3}\right) \delta \mathrm{ppm} 8.72(\mathrm{~s}, 1 \mathrm{H}), 7.87(\mathrm{~d}, J=7.8 \mathrm{~Hz}), 7.76(\mathrm{ddd}, J=8.4,7.0,1.3 \mathrm{~Hz}, 1 \mathrm{H}), 7.60$ $(\mathrm{ddd}, J=8.2,7.0,1.2 \mathrm{~Hz}, 1 \mathrm{H}), 7.39-7.35(\mathrm{~m}, 1 \mathrm{H}), 7.22-7.19(\mathrm{~m}, 1 \mathrm{H}), 7.16-7.12(\mathrm{~m}, 1 \mathrm{H}), 5.83$ (br.s, $1 \mathrm{H}), 3.97(\mathrm{dt}, J=6.9,5.9 \mathrm{~Hz}, 2 \mathrm{H}), 3.09(\mathrm{t}, J=6.9 \mathrm{~Hz}, 2 \mathrm{H}) .{ }^{13} \mathrm{C}\left(125 \mathrm{MHz}, \mathrm{CDCl}_{3}\right) \delta$ ppm 159.3, 155.4, 149.5 (q, $J=1.8 \mathrm{~Hz}), 141.3,132.6,130.1,128.7,127.3,126.1,121.4,120.5$ $(\mathrm{q}, J=257.2 \mathrm{~Hz}), 120.2,119.1,114.9,42.1,35.1 .{ }^{19} \mathrm{~F}\left(470 \mathrm{MHz}, \mathrm{CDCl}_{3}\right) \delta \mathrm{ppm}-57.7(\mathrm{~s}, 3 \mathrm{~F})$. HRMS (EI), $\mathrm{M}+1$ calculated for $\mathrm{C}_{17} \mathrm{H}_{14} \mathrm{~F}_{3} \mathrm{~N}_{3} \mathrm{O}, 306.1965$, found 306.1967 .

[2-(3-Ethoxyphenyl)ethyl]quinazolin-4-ylamine, 14a

Following general procedure A, using 4-chloroquinazoline (5, $151 \mathrm{mg}, 0.92 \mathrm{mmol}), 3$ ethoxyphenylethylamine $(155 \mathrm{mg}, 0.92 \mathrm{mmol})$ and potassium carbonate $(127 \mathrm{mg}, 0.92 \mathrm{mmol})$ gave 14a as off-white crystals $(211 \mathrm{mg}, 78 \%)$. mp $148.5-148.8{ }^{\circ} \mathrm{C} ;{ }^{1} \mathrm{H}\left(500 \mathrm{MHz}, \mathrm{CDCl}_{3}\right)$ $\delta$ ppm $8.71(\mathrm{~s}, 1 \mathrm{H}), 7.86(\mathrm{~d}, J=8.3 \mathrm{~Hz}, 1 \mathrm{H}), 7.74(\mathrm{ddd}, J=8.3,7.0,1.2 \mathrm{~Hz}, 1 \mathrm{H}), 7.56(\mathrm{~d}$, 
$J=8.0 \mathrm{~Hz}, 1 \mathrm{H}), 7.44(\mathrm{ddd}, J=8.0,7.0,1.1 \mathrm{~Hz}, 1 \mathrm{H}), 6.86(\mathrm{~d}, J=7.5 \mathrm{~Hz}, 1 \mathrm{H}), 6.83-6.79(\mathrm{~m}, 2 \mathrm{H})$, 5.79 (br.s, $1 \mathrm{H}), 4.02(\mathrm{q}, J=7.0 \mathrm{~Hz}, 2 \mathrm{H}), 3.96(\mathrm{dt}, J=6.7,5.8 \mathrm{~Hz}, 2 \mathrm{H}), 1.42(\mathrm{t}, J=7.0 \mathrm{~Hz}, 3 \mathrm{H})$. ${ }^{13} \mathrm{C}\left(125 \mathrm{MHz}, \mathrm{CDCl}_{3}\right) \delta$ ppm 159.4, 159.3, 155.5, 149.5, 140.4, 132.5, 129.8, 128.7, 126.0, 121.0, 120.3, 115.1, 115.0, 112.6, 63.4, 42.1, 35.3, 14.8. HRMS (EI), $\mathrm{M}+1$ calculated for $\mathrm{C}_{18} \mathrm{H}_{19} \mathrm{~N}_{3} \mathrm{O}$, 294.1601, found 294.1616.

Quinazolin-4-yl\{2-[2-(trifluromethyl)phenyl]ethyl\}amine, 15a

Following general procedure A outlined, using 4-chloroquinazoline $(5,100 \mathrm{mg}, 0.59 \mathrm{mmol})$, 2-(2-trifluoromethylphenyl)ethylamine $(117 \mathrm{mg}, 0.59 \mathrm{mmol})$ and potassium carbonate (81 mg, $0.59 \mathrm{mmol}$ ) gave $15 \mathrm{a}$ as a yellow flaky solid $(68 \mathrm{mg}, 34 \%) . \mathrm{mp} 134.4-137.4{ }^{\circ} \mathrm{C} ;{ }^{1} \mathrm{H}$ $\left(500 \mathrm{MHz}, \mathrm{CDCl}_{3}\right) \delta \mathrm{ppm} 8.66(\mathrm{~s}, 1 \mathrm{H}), 8.12-8.05(\mathrm{~m}, 1 \mathrm{H}), 7.95(\mathrm{~d}, J=8.3 \mathrm{~Hz}, 1 \mathrm{H}), 7.80-7.74$ $(\mathrm{m}, 1 \mathrm{H}), 7.66(\mathrm{~d}, J=7.8 \mathrm{~Hz}, 1 \mathrm{H}), 7.54-7.49(\mathrm{~m}, 1 \mathrm{H}), 7.49-7.46(\mathrm{~m}, 1 \mathrm{H}), 7.37-7.32(\mathrm{~m}, 1 \mathrm{H}), 4.04$ $(\mathrm{dt}, J=6.9,6.7 \mathrm{~Hz}, 2 \mathrm{H}), 3.30(\mathrm{t}, J=7.2 \mathrm{~Hz}, 2 \mathrm{H}) .{ }^{13} \mathrm{C}\left(125 \mathrm{MHz}, \mathrm{CDCl}_{3}\right) \delta \mathrm{ppm} 159.9,153.5$, $145.7,137.3,133.6,132.0,131.7,128.9(\mathrm{q}, J=29.7 \mathrm{~Hz}), 126.9,126.1(\mathrm{q}, J=5.7 \mathrm{~Hz}), 126.8,125.7$, $124.5(\mathrm{q}, J=254.0 \mathrm{~Hz}), 121.9,114.4,42.7,31.8 .{ }^{19} \mathrm{~F}\left(470 \mathrm{MHz}, \mathrm{CDCl}_{3}\right) \delta \mathrm{ppm}-59.2(\mathrm{~s}, 3 \mathrm{~F})$ HRMS (EI), $\mathrm{M}+1$ calculated for $\mathrm{C}_{17} \mathrm{H}_{14} \mathrm{~F}_{3} \mathrm{~N}_{3}, 318.1213$, found 318.1220. This compound appears in referenced patent and fungicidal activity was reported [17].

(6-Fluoroquinazolin-4-yl)\{2-[4-(trifluormethoxy)phenyl]ethyl\}amine, 16a

Following general procedure A, using 4-chloro-6-fluoroquinazoline (16, $100 \mathrm{mg}$, $0.55 \mathrm{mmol}), 2$-[4-(trifluromethoxy)phenyl]ethylamine (112 $\mathrm{mg}, 0.55 \mathrm{mmol})$, and potassium carbonate $(76 \mathrm{mg}, 0.55 \mathrm{mmol})$ gave $16 \mathrm{a}$ as a white solid $(99 \mathrm{mg}, 51 \%) . \mathrm{mp} 171.6-172.6{ }^{\circ} \mathrm{C}$; ${ }^{1} \mathrm{H}\left(500 \mathrm{MHz}, \mathrm{CDCl}_{3}\right) \delta \mathrm{ppm} 8.70(\mathrm{~s}, 1 \mathrm{H}), 7.89(\mathrm{dd}, J=9.1,5.3 \mathrm{~Hz}, 1 \mathrm{H}), 7.52$ (ddd, 9.1, 8.2, $2.7 \mathrm{~Hz}, 1 \mathrm{H}), 7.32-7.29(\mathrm{~m}, 2 \mathrm{H}), 7.23-7.19(\mathrm{~m}, 3 \mathrm{H}), 5.57$ (br.s, $1 \mathrm{H}), 3.96(\mathrm{dt}, J=7.0,5.8 \mathrm{~Hz}, 2 \mathrm{H})$, $3.08(\mathrm{t}, J=7.0 \mathrm{~Hz}, 2 \mathrm{H}) .{ }^{13} \mathrm{C}\left(125 \mathrm{MHz}, \mathrm{CDCl}_{3}\right) \delta \mathrm{ppm} 160.0(\mathrm{~d}, J=248.9 \mathrm{~Hz}), 159.0,148.1(\mathrm{q}$, $J=1.9 \mathrm{~Hz}), 146.5,137.5,131.4(\mathrm{~d}, J=8.4 \mathrm{~Hz}), 130.1,122.2(\mathrm{~d}, J=24.4 \mathrm{~Hz}), 121.5,121.3,120.5$ $(\mathrm{q}, J=255.5 \mathrm{~Hz}), 115.2(\mathrm{~d}, J=7.9 \mathrm{~Hz}), 104.7(\mathrm{~d}, J=22.7 \mathrm{~Hz}), 42.4,34.6 .{ }^{19} \mathrm{~F}\left(470 \mathrm{MHz}, \mathrm{CDCl}_{3}\right)$ $\delta$ ppm $-57.9(\mathrm{~s}, 3 \mathrm{~F}),-112.3(\mathrm{ddd}, J=5.4,8.5,8.5 \mathrm{~Hz}, 1 \mathrm{~F})$. HRMS (EI), $\mathrm{M}+1$ calculated for $\mathrm{C}_{17} \mathrm{H}_{13} \mathrm{~F}_{4} \mathrm{~N}_{3} \mathrm{O}, 352.1068$, found 352.1078 . This compound was shown to inhibit autophagy in referenced patent [22].

(6-Methylquinazolin-4-yl)\{2-[4-(trifluormethoxy)phenyl]ethyl\}amine, 17a

Following general procedure A, using 4-chloro-6-methylquinazoline (17, $100 \mathrm{mg}$, $0.56 \mathrm{mmol}), 2$-[4-(trifluoromethoxy)phenyl]ethylamine $(115 \mathrm{mg}, 0.56 \mathrm{mmol})$, and potassium carbonate $(77 \mathrm{mg}, 0.56 \mathrm{mmol})$ gave $\mathbf{1 7 a}$ as an off-white solid $(134 \mathrm{mg}, 69 \%)$ mp 134.3 $135.0^{\circ} \mathrm{C} ;{ }^{1} \mathrm{H}\left(500 \mathrm{MHz}, \mathrm{CDCl}_{3}\right) \delta \mathrm{ppm} 8.67(\mathrm{~s}, 1 \mathrm{H}), 7.77(\mathrm{~d}, J=8.5 \mathrm{~Hz}, 1 \mathrm{H}), 7.58(\mathrm{dd}, J=8.5$, $1.6 \mathrm{~Hz}, 1 \mathrm{H}), 7.36(\mathrm{~s}, 1 \mathrm{H}), 7.31-7.27(\mathrm{~m}, 2 \mathrm{H}), 5.76$ (br.s, $1 \mathrm{H}), 3.94(\mathrm{dt}, J=6.9,5.9 \mathrm{~Hz}, 2 \mathrm{H})$, $3.08(\mathrm{t}, J=7.0 \mathrm{~Hz}, 2 \mathrm{H}), 2.50(\mathrm{~s}, 3 \mathrm{H}) .{ }^{13} \mathrm{C}\left(125 \mathrm{MHz}, \mathrm{CDCl}_{3}\right) \delta \mathrm{ppm} 158.9,154.6,148.0(\mathrm{q}$, $J=1.8 \mathrm{~Hz}), 147.8,137.8,136.1,134.5,130.2,128.5,121.2,120.5(\mathrm{q}, J=256.9 \mathrm{~Hz}), 119.4,114.8$, 42.3, 34.7, 21.7. ${ }^{19} \mathrm{~F}\left(470 \mathrm{MHz}, \mathrm{CDCl}_{3}\right) \delta \mathrm{ppm}-57.9$ (s, 3F). HRMS (EI), M + 1 calculated for $\mathrm{C}_{18} \mathrm{H}_{16} \mathrm{~F}_{3} \mathrm{~N}_{3} \mathrm{O}$, 348.1318, found 348.1336.

(6-Methoxyquinazolin-4-yl)\{2-[4-(trifluormethoxy)phenyl]ethyl\}amine, 18a

Following general procedure A, using 4-chloro-6-methoxyquinazoline (18, $100 \mathrm{mg}$, $0.51 \mathrm{mmol}), 2$-[4-(trifluoromethoxy)phenyl]ethylamine ( $105 \mathrm{mg}, 0.51 \mathrm{mmol})$, and potassium carbonate $(71 \mathrm{mg}, 0.51 \mathrm{mmol})$ gave $18 \mathrm{a}$ as a white solid $(93 \mathrm{mg}, 50 \%)$. mp $144.7-145.2{ }^{\circ} \mathrm{C}$; ${ }^{1} \mathrm{H}\left(500 \mathrm{MHz}, \mathrm{CDCl}_{3}\right) \delta \mathrm{ppm} 8.64(\mathrm{~s}, 1 \mathrm{H}), 7.81(\mathrm{~d}, J=9.1 \mathrm{~Hz}, 1 \mathrm{H}), 7.41(\mathrm{dd}, J=9.1,2.5 \mathrm{~Hz}$, $1 \mathrm{~Hz}), 7.32-7.26(\mathrm{~m}, 2 \mathrm{H}), 7.20(\mathrm{~d}, J=8.1 \mathrm{~Hz}, 2 \mathrm{H}), 6.82(\mathrm{~d}, J=2.5 \mathrm{~Hz}, 1 \mathrm{H}), 5.65$ (br.s, $1 \mathrm{H})$, $3.95(\mathrm{dt}, J=6.8,6.2 \mathrm{~Hz}, 2 \mathrm{H}), 3.87(\mathrm{~s}, 3 \mathrm{H}), 3.08(\mathrm{t}, J=7.0 \mathrm{~Hz}, 2 \mathrm{H}) .{ }^{13} \mathrm{C}\left(125 \mathrm{MHz}, \mathrm{CDCl}_{3}\right) \delta$ ppm 158.6, 157.6, 153.4, $148.0(\mathrm{q}, J=1.8 \mathrm{~Hz}), 144.9,137.9,130.3,130.2,123.6,121.2,120.5(\mathrm{q}$, $J=258.4 \mathrm{~Hz}), 115.3,99.8,55.6,42.3,34.7 .{ }^{19} \mathrm{~F}\left(470 \mathrm{MHz}, \mathrm{CDCl}_{3}\right) \delta \mathrm{ppm}-57.9$ (s, 3F). HRMS (EI), $\mathrm{M}+1$ calculated for $\mathrm{C}_{18} \mathrm{H}_{16} \mathrm{~F}_{3} \mathrm{~N}_{3} \mathrm{O}_{2}, 364.1267$, found 364.1276.

(7-Fluoroquinazolin-4-yl)\{2-[4-(trifluormethoxy)phenyl]ethyl\}amine, 19a 
Following general procedure A, using 4-chloro-7-fluoroquinazoline (19, $100 \mathrm{mg}$, $0.55 \mathrm{mmol}), 2$-[4-(trifluoromethoxy)phenyl]ethylamine $(112 \mathrm{mg}, 0.55 \mathrm{mmol})$, and potassium carbonate $(76 \mathrm{mg}, 0.55 \mathrm{~mol})$ gave $19 \mathrm{a}$ as off-white crystals $(107 \mathrm{mg}, 56 \%) . \mathrm{mp} 128.9-129.7^{\circ} \mathrm{C}$; ${ }^{1} \mathrm{H}\left(500 \mathrm{MHz}, \mathrm{CDCl}_{3}\right) \delta \mathrm{ppm} 8.69(\mathrm{~s}, 1 \mathrm{H}), 7.60(\mathrm{dd}, J=9.1,5.6 \mathrm{~Hz}, 1 \mathrm{H}), 7.49(\mathrm{dd}, J=9.8$, $2.5 \mathrm{~Hz}, 1 \mathrm{H}), 7.32-7.26(\mathrm{~m}, 2 \mathrm{H}), 7.24-7.17(\mathrm{~m}, 3 \mathrm{H}), 5.74$ (br.s, $1 \mathrm{H}), 3.96(\mathrm{dt}, J=6.9,5.9 \mathrm{~Hz}$, 2H), $3.07(\mathrm{t}, J=7.0 \mathrm{~Hz}, 2 \mathrm{H}) .{ }^{13} \mathrm{C}\left(125 \mathrm{MHz}, \mathrm{CDCl}_{3}\right) \delta \mathrm{ppm} 165.0(\mathrm{~d}, J=253.3 \mathrm{~Hz}), 159.1$, $156.4,151.6(\mathrm{~d}, J=13.1 \mathrm{~Hz}), 148.1(\mathrm{q}, J=2.0 \mathrm{~Hz}), 137.6,130.1,122.8(\mathrm{~d}, J=10.4 \mathrm{~Hz}), 121.3$, $120.5(\mathrm{q}, J=256.9 \mathrm{~Hz}), 115.8(\mathrm{~d}, J=24.9 \mathrm{~Hz}), 112.9(\mathrm{~d}, J=20.4 \mathrm{~Hz}), 111.8,42.3,34.6 .{ }^{19} \mathrm{~F}$ $\left(470 \mathrm{MHz}, \mathrm{CDCl}_{3}\right) \delta \mathrm{ppm}-57.9(\mathrm{~s}, 3 \mathrm{~F}),-105.3$ (ddd, $\left.J=5.3,9.2,9.2 \mathrm{~Hz}, 1 \mathrm{~F}\right)$. HRMS (EI), M + 1 calculated for $\mathrm{C}_{17} \mathrm{H}_{13} \mathrm{~F}_{4} \mathrm{~N}_{3} \mathrm{O}$, 352.1068, found 352.1072.

(7-Chloroquinazolin-4-yl)\{2-[4-(trifluormethoxy)phenyl]ethyl\}amine, 20a

Following general procedure A, using 4,7-dichloroquinazoline $(\mathbf{2 0}, 100 \mathrm{mg}, 0.51 \mathrm{mmol})$, 2-[4-(trifluoromethoxy)phenyl]ethylamine $(105 \mathrm{mg}, 0.51 \mathrm{mmol})$, and potassium carbonate (78 mg, $0.56 \mathrm{~mol}$ ) gave $20 \mathrm{a}$ as off-white flaky crystals $(72 \mathrm{mg}, 50 \%) . \mathrm{mp} 153.0-153.4{ }^{\circ} \mathrm{C} ;{ }^{1} \mathrm{H}$ $(500 \mathrm{MHz}, \mathrm{MeOD}) \delta \mathrm{ppm} 8.47(\mathrm{~s}, 1 \mathrm{H}), 8.03(\mathrm{~d}, J=8.6 \mathrm{~Hz}, 1 \mathrm{H}), 7.69(\mathrm{~d}, J=2.1 \mathrm{~Hz}, 1 \mathrm{H}), 7.49$ $(\mathrm{dd}, J=8.6,2.1 \mathrm{~Hz}, 1 \mathrm{H}), 7.36(\mathrm{dt}, J=8.6,2.7 \mathrm{~Hz}, 2 \mathrm{H}), 7.21-7.16(\mathrm{~m}, 2 \mathrm{H}), 3.86(\mathrm{t}, J=7.3 \mathrm{~Hz}$, $2 \mathrm{H}), 3.06(\mathrm{t}, J=7.3 \mathrm{~Hz}, 2 \mathrm{H}) .{ }^{13} \mathrm{C}(125 \mathrm{MHz}, \mathrm{MeOD}) \delta \mathrm{ppm} \mathrm{159.8,} \mathrm{155.8,} \mathrm{149.3,} 147.7(\mathrm{q}$, $J=1.7 \mathrm{~Hz}), 138.6,130.1,126.4,125.4,123.9,120.7,120.5(\mathrm{q}, J=255.1 \mathrm{~Hz}), 113.6,42.2,33.9 .{ }^{19} \mathrm{~F}$ (470 MHz, MeOD) $\delta$ ppm -59.6 (s, 3F). HRMS (EI), $\mathrm{M}+1$ calculated for $\mathrm{C}_{17} \mathrm{H}_{13} \mathrm{ClF}_{3} \mathrm{~N}_{3} \mathrm{O}$, 368.0772, found 368.0767.

(7-Bromoquinazolin-4-yl)\{2-[4-(trifluormethoxy)phenyl]ethyl\}amine, 21a

Following general procedure A, using 4-chloro-7-bromoquinazoline $(\mathbf{2 1}, 75 \mathrm{mg}, 0.30 \mathrm{mmol})$, 2-[4-(trifluoromethoxy)phenyl]ethylamine $(62 \mathrm{mg}, 0.30 \mathrm{mmol})$, and potassium carbonate (41 mg, $0.30 \mathrm{mmol}$ ) gave $21 \mathrm{a}$ as off-white crystals $(52 \mathrm{mg}, 42 \%) . \mathrm{mp} 151.8-152.8{ }^{\circ} \mathrm{C} ;{ }^{1} \mathrm{H}$ $\left(500 \mathrm{MHz}, \mathrm{CDCl}_{3}\right) \delta \mathrm{ppm} 8.68(\mathrm{~s}, 1 \mathrm{H}), 8.06(\mathrm{~s}, 1 \mathrm{H}), 7.56(\mathrm{~s}, 2 \mathrm{H}), 7.32-7.26(\mathrm{~m}, 2 \mathrm{H}), 7.20(\mathrm{~d}$, $J=8.0 \mathrm{~Hz}, 2 \mathrm{H}), 6.18$ (br.s, $1 \mathrm{H}), 3.97(\mathrm{dt}, J=6.9,6.1 \mathrm{~Hz}, 2 \mathrm{H}), 3.09(\mathrm{t}, J=7.0 \mathrm{~Hz}, 2 \mathrm{H}) .{ }^{13} \mathrm{C}$ $\left(125 \mathrm{MHz}, \mathrm{CDCl}_{3}\right) \delta \mathrm{ppm} 159.4,155.7,149.4,148.1(\mathrm{q}, J=1.6 \mathrm{~Hz}), 137.4,130.4,130.1,129.7$, $127.4,122.2,121.3,120.5(\mathrm{q}, J=257.0 \mathrm{~Hz}), 113.4,42.5,34.5 .{ }^{19} \mathrm{~F}\left(470 \mathrm{MHz}, \mathrm{CDCl}_{3}\right) \delta \mathrm{ppm}$ $-57.9(\mathrm{~s}, 3 \mathrm{~F})$. HRMS (EI), $\mathrm{M}+1$ calculated for $\mathrm{C}_{17} \mathrm{H}_{13} \mathrm{BrF}_{3} \mathrm{~N}_{3} \mathrm{O}, 412.0267$, found 412.0246 .

\{2-[4-(Trifluoromethoxy)phenyl]ethyl\}[7-(trifluoromethyl)quinazolin-4-yl]amine, 22a

Following general procedure A, using 7-(trifluoromethyl)-4-chloroquinazoline (22, $100 \mathrm{mg}, 0.45 \mathrm{mmol}$ ), 2-[4-(trifluoromethoxy)phenyl]ethylamine ( $93 \mathrm{mg}, 0.45 \mathrm{mmol}$ ), and potassium carbonate $(69 \mathrm{mg}, 0.50 \mathrm{~mol})$ gave $22 \mathrm{a}$ as off-white crystals $(82 \mathrm{mg}, 55 \%) . \mathrm{mp}$ 149.7-152.3 ${ }^{\circ} \mathrm{C} ;{ }^{1} \mathrm{H}(500 \mathrm{MHz}, \mathrm{MeOD}) \delta \mathrm{ppm} 8.44(\mathrm{~d}, J=8.4 \mathrm{~Hz}, 1 \mathrm{H}), 8.41-8.37(\mathrm{~m}, 1 \mathrm{H})$, $8.01(\mathrm{~s}, 1 \mathrm{H}), 7.86(\mathrm{dd}, J=8.4,1.5 \mathrm{~Hz}, 1 \mathrm{H}), 7.42(\mathrm{dt}, J=8.6,2.8 \mathrm{~Hz}, 2 \mathrm{H}), 7.31-7.25(\mathrm{~m}, 2 \mathrm{H})$, $3.22(\mathrm{t}, J=7.7 \mathrm{~Hz}, 2 \mathrm{H}), 3.02(\mathrm{t}, J=7.7 \mathrm{~Hz}, 2 \mathrm{H}) .{ }^{13} \mathrm{C}(125 \mathrm{MHz}, \mathrm{MeOD}) \delta \mathrm{ppm} 160.4,148.3$ $(\mathrm{q}, J=1.8 \mathrm{~Hz}), 147.3,135.8,135.8(\mathrm{q}, J=32.9 \mathrm{~Hz}), 130.2,127.7,125.0,123.4(\mathrm{q}, J=272.4 \mathrm{~Hz})$, $123.1(\mathrm{q}, J=3.6 \mathrm{~Hz}), 122.8(\mathrm{~m}), 121.2,120.5(\mathrm{q}, J=255.5 \mathrm{~Hz}), 119.5,40.3,32.4 .{ }^{19} \mathrm{~F}(470 \mathrm{MHz}$, MeOD) $\delta$ ppm $-59.6(\mathrm{~s}, 3 \mathrm{~F}),-64.8(\mathrm{~s}, 3 \mathrm{~F})$. HRMS (EI), $\mathrm{M}+1$ calculated for $\mathrm{C}_{18} \mathrm{H}_{13} \mathrm{~F}_{6} \mathrm{~N}_{3} \mathrm{O}$, 402.1036, found 402.1025.

(7-Methoxyquinazolin-4-yl)\{2-[4-(trifluormethoxy)phenyl]ethyl\}amine, 23a

Following general procedure A, using 7-(methoxy)-4-chloroquinazoline (23, $100 \mathrm{mg}$, $0.54 \mathrm{mmol}), 2$-[4-(trifluoromethoxy)phenyl]ethylamine $(110 \mathrm{mg}, 0.54 \mathrm{mmol})$, and potassium carbonate $(82 \mathrm{mg}, 0.59 \mathrm{~mol})$ gave $23 \mathrm{a}$ as colorless crystals $(68 \mathrm{mg}, 51 \%) . \mathrm{mp} 158.0-159.0^{\circ} \mathrm{C}$; ${ }^{1} \mathrm{H}(500 \mathrm{MHz}, \mathrm{MeOD}) \delta \mathrm{ppm} 8.40(\mathrm{~s}, 1 \mathrm{H}), 7.94(\mathrm{~d}, J=9.1 \mathrm{~Hz}, 1 \mathrm{H}), 7.36(\mathrm{dt}, J=8.7,2.8 \mathrm{~Hz}$, $2 \mathrm{H}), 7.21-7.16(\mathrm{~m}, 2 \mathrm{H}), 7.11(\mathrm{dd}, J=9.1,2.6 \mathrm{~Hz}, 1 \mathrm{H}), 7.08(\mathrm{~d}, J=2.5 \mathrm{~Hz}, 1 \mathrm{H}), 3.93(\mathrm{~s}, 3 \mathrm{H})$, $3.83(\mathrm{t}, J=7.4 \mathrm{~Hz}, 2 \mathrm{H}), 3.05(\mathrm{t}, J=7.4 \mathrm{~Hz}, 2 \mathrm{H}) .{ }^{13} \mathrm{C}(125 \mathrm{MHz}, \mathrm{MeOD}) \delta \mathrm{ppm} 163.5,159.6$, 155.0, 150.6, $147.7(\mathrm{q}, J=1.7 \mathrm{~Hz}), 138.7,130.1,123.5,120.7,120.6(\mathrm{q}, J=255.1 \mathrm{~Hz}), 117.3$, 109.1, 105.5, 54.7, 42.1, 34.1. ${ }^{19} \mathrm{~F}(470 \mathrm{MHz}, \mathrm{MeOD}) \delta \mathrm{ppm}-59.5$ (3F). HRMS (EI), $\mathrm{M}+1$ calculated for $\mathrm{C}_{18} \mathrm{H}_{16} \mathrm{~F}_{3} \mathrm{~N}_{3} \mathrm{O}_{2}, 364.1267$, found 364.1259. 
(2-Methylquinazolin-4-yl)\{2-[4-(trifluormethoxy)phenyl]ethyl\}amine, 24a

Following general procedure A, using 4-chloro-2-methylquinazoline (24, $100 \mathrm{mg}$, $0.56 \mathrm{mmol}), 2$-[4-(trifluoromethoxy)phenyl]ethylamine ( $115 \mathrm{mg}, 0.56 \mathrm{mmol})$, and potassium carbonate $(77 \mathrm{mg}, 0.56 \mathrm{mmol})$ gave $24 \mathrm{a}$ as a pale yellow solid $(153 \mathrm{mg}, 79 \%)$. mp 124.4$127.2{ }^{\circ} \mathrm{C} ;{ }^{1} \mathrm{H}\left(500 \mathrm{MHz}, \mathrm{CDCl}_{3}\right) \delta \mathrm{ppm} 7.81(\mathrm{~d}, J=8.3 \mathrm{~Hz}, 1 \mathrm{H}), 7.74-7.68(\mathrm{~m}, 1 \mathrm{H}), 7.60(\mathrm{~d}$, $J=8.1 \mathrm{~Hz}, \mathrm{H}), 7.42-7.37(\mathrm{~m}, 2 \mathrm{H}), 7.20(\mathrm{~d}, J=8.0 \mathrm{~Hz}, 2 \mathrm{H}), 5.91$ (br.s, $1 \mathrm{H}), 3.96(\mathrm{dt}, J=6.9$, $6.0 \mathrm{~Hz}, 2 \mathrm{H}), 3.07(\mathrm{t}, J=7.0 \mathrm{~Hz}, 2 \mathrm{H}), 2.69(\mathrm{~s}, 3 \mathrm{H}) .{ }^{13} \mathrm{C}\left(125 \mathrm{MHz}, \mathrm{CDCl}_{3}\right) \delta \mathrm{ppm} 164.3,159.3$, $149.4,148.0(\mathrm{q}, J=1.7 \mathrm{~Hz}), 137.8,132.7,130.2,127.5,125.3,121.2,120.5(\mathrm{q}, J=256.9 \mathrm{~Hz})$, $120.3,112.8,42.2,34.7,26.5 .{ }^{19} \mathrm{~F}\left(470 \mathrm{MHz}, \mathrm{CDCl}_{3}\right) \delta \mathrm{ppm}-57.9$ (s, 3F). HRMS (EI), M + 1 calculated for $\mathrm{C}_{18} \mathrm{H}_{16} \mathrm{~F}_{3} \mathrm{~N}_{3} \mathrm{O}, 348.1318$, found 348.1335 .

(2-Cyclopropylquinazolin-4-yl)\{2-[4-(trifluoromethoxy)phenyl]ethyl\}amine, 25a

Following general procedure A, using 4-chloro-2-cyclopropylquinazolne $(\mathbf{2 5}, 100 \mathrm{mg}$, $0.49 \mathrm{mmol}), 2-[4$-(trifluoromethoxy)phenyl]ethylamine $(100 \mathrm{mg}, 0.49 \mathrm{mmol})$ and potassium carbonate $\left(67 \mathrm{mg}, 0.48 \mathrm{mmol}\right.$ ) gave $25 \mathrm{a}$ as a tan solid $(119 \mathrm{mg}, 65 \%) . \mathrm{mp} 205.1-205.5^{\circ} \mathrm{C} ;{ }^{1} \mathrm{H}$ $(500 \mathrm{MHz}, \mathrm{MeOD}) \delta \mathrm{ppm} 8.24(\mathrm{~d}, J=8.5 \mathrm{~Hz}, 1 \mathrm{H}), 8.02-7.97(\mathrm{~m}, 1 \mathrm{H}), 7.74-7.68(\mathrm{~m}, 2 \mathrm{H}), 7.36$ $(\mathrm{dt}, J=8.6,2.7 \mathrm{~Hz}, 2 \mathrm{H}), 7.20(\mathrm{~d}, J=7.8 \mathrm{~Hz}, 2 \mathrm{H}), 3.99(\mathrm{t}, J=7.1 \mathrm{~Hz}, 2 \mathrm{H}), 3.08(\mathrm{t}, J=7.1 \mathrm{~Hz}$, 2H), 2.23-2.16 (m, 1H), 1.44-1.32 (m, 4H). ${ }^{13} \mathrm{C}(125 \mathrm{MHz}, \mathrm{MeOD}) \delta \mathrm{ppm} 166.5,160.8,147.9$, $138.3,137.9,135.7,130.2,127.7,123.3,120.9,120.5(\mathrm{q}, J=255.2 \mathrm{~Hz}), 118.0,111.9,42.9,33.8$, 14.5, 11.2. ${ }^{19} \mathrm{~F}(470 \mathrm{MHz}, \mathrm{MeOD}) \delta \mathrm{ppm}-59.6$ (s, 3F). HRMS (EI), $\mathrm{M}+1$ calculated for $\mathrm{C}_{20} \mathrm{H}_{18} \mathrm{~F}_{3} \mathrm{~N}_{3} \mathrm{O}$, 374.1475, found 374.1493.

(7-Fluoro-2-methylquinazolin-4-yl)\{2-[4-(trifluormethoxy)phenyl]ethyl\}amine, 26a

Following general procedure A, using 4-chloro-7-fluoro-2-methylquinazoline (26, $75 \mathrm{mg}, 0.37 \mathrm{mmol}), 2$-[4-(trifluoromethoxy)phenyl]ethylamine $(77 \mathrm{mg}, 0.37 \mathrm{mmol})$, and potassium carbonate $(52 \mathrm{mg}, 0.37 \mathrm{mmol}$ ) gave $26 \mathbf{a}$ as a white solid $(58 \mathrm{mg}, 43 \%)$. $\mathrm{mp} 121.7-$ $124.7^{\circ} \mathrm{C} ;{ }^{1} \mathrm{H}\left(500 \mathrm{MHz}, \mathrm{CDCl}_{3}\right) \delta \mathrm{ppm} 14.78(\mathrm{~s}, 1 \mathrm{H}), 10.62(\mathrm{~s}, 1 \mathrm{H}), 9.10(\mathrm{dd}, J=9.1,5.2 \mathrm{~Hz}$, $1 \mathrm{H}), 7.84(\mathrm{dd}, J=8.6,2.4 \mathrm{~Hz}, 1 \mathrm{H}), 7.30-7.22(\mathrm{~m}, 1 \mathrm{H}), 7.19-7.13(\mathrm{~m}, 2 \mathrm{H}), 7.06-7.02(\mathrm{~m}, 2 \mathrm{H})$, $4.14(\mathrm{q}, J=6.9 \mathrm{~Hz}, 2 \mathrm{H}), 3.22(\mathrm{t}, J=7.4 \mathrm{~Hz}, 2 \mathrm{H}), 2.77(\mathrm{~s}, 3 \mathrm{H}) .{ }^{13} \mathrm{C}\left(125 \mathrm{MHz}, \mathrm{CDCl}_{3}\right) \delta \mathrm{ppm}$ $166.1(\mathrm{~d}, J=259.4 \mathrm{~Hz}), 162.0,160.0,149.3(\mathrm{q}, J=2.0 \mathrm{~Hz}), 140.7,140.5(\mathrm{~d}, J=12.9 \mathrm{~Hz}), 129.8$, $128.7(\mathrm{~d}, J=10.3 \mathrm{~Hz}), 127.4,121.4,120.4(\mathrm{q}, J=257.1 \mathrm{~Hz}), 119.1,116.9(\mathrm{~d}, J=23.9 \mathrm{~Hz}), 108.6$, $104.7(\mathrm{~d}, J=25.6 \mathrm{~Hz}), 43.1,34.6,22.4 .{ }^{19} \mathrm{~F}\left(470 \mathrm{MHz}, \mathrm{CDCl}_{3}\right) \delta \mathrm{ppm}-57.8(\mathrm{~s}, 3 \mathrm{~F}),-98.3$ (ddd, $J=4.8,8.1,8.1 \mathrm{~Hz}, 1 \mathrm{~F}$ ). HRMS (EI), $\mathrm{M}+1$ calculated for $\mathrm{C}_{18} \mathrm{H}_{15} \mathrm{~F}_{4} \mathrm{~N}_{3} \mathrm{O}, 366.1224$, found 366.1199 .

\subsection{Biological Assessments}

Mycobacterium bovis BCG and Mycobacterium tuberculosis strains were routinely cultured in Middlebrook 7H9 medium (Becton Dickson and Company Limited, Franklin Lakes, NJ, USA) supplemented with $0.05 \%$ tween $80,0.5 \%$ glycerol, bovine serum albumin fraction $\mathrm{V}$, D-glucose, and $\mathrm{NaCl}$. M. tuberculosis clinical isolate N0145 was a gift from Sebastien Gagneux (Swiss Tropical and Public Health Institute at the University of Basel). Prior to use, bacteria cells were pelleted and resuspended in medium without glycerol and adjusted to a cell density of circa $10^{7} \mathrm{CFU} / \mathrm{mL}$. The test compounds were tested in the presence or absence of Q203. Prior to the assay, Q203 or DMSO (solvent control) was added to the bacteria cultures. Q203 was used in excess (at $100 \mathrm{nM}$ ) to completely inhibit the function of the cytochrome $b c c: a a_{3}$, thereby revealing the activity of cyt-bd in the assay. The Q203 ATP IC 50 for M. bovis BCG, M. tuberculosis H37Rv, and M. tuberculosis N0145 are 2.6, 1.0 , and $2.5 \mathrm{nM}$, respectively (Supplementary Materials Figure S61). The test compounds were tested in eight concentrations, starting at $25 \mu \mathrm{M}$, in two-fold dilutions $(0.2-25.0 \mu \mathrm{M})$ in the presence or absence of $100 \mathrm{nM}$ Q203. $1 \mu \mathrm{L}$ of test compounds of varying concentrations was added to each well of 96-well white plates, and $100 \mu \mathrm{L}$ of bacterial culture was subsequently added. The assay plates were incubated at $37^{\circ} \mathrm{C}$ for $15 \mathrm{~h}$, after which the BacTiter-Glo ${ }^{\mathrm{TM}}$ (Promega, Madison, WI, USA) reagent was added. Following a 12-min 
incubation, the luminescence of each plate was measured using a BioTek Cytation 3 Cell Imaging Multiple mode reader. $\mathrm{IC}_{50}$ values were determined using GraphPad Prism 9.

\section{Results and Discussion}

Our initial efforts were to probe the effect of alteration and modification of the phenethylaniline moiety around a fixed quinazoline core. This was done by syntheses of 10 compounds and screening against M. bovis BCG and Mtb strains (H37Rv and N0145) to assess activity against mycobacterial cyt- $b d$ (Table 1 ). A representative compound from this set (7a) served as the foundation toward the design of a second set of 11 compounds that focused on modification of the quinazoline core and were subsequently screened for cyt- $b d$ activity (Table 2).

Table 1. In vitro activity of phenethyl-quinazolin-4-yl-amines (3, 6a-15a) and control compounds bedaquiline (BDQ), ND11992 (2), and aurachin D against three mycobacterial strains (M. bovis BCG, M. tuberculosis H37Rv, and M. tuberculosis N0145).

\begin{tabular}{|c|c|c|c|c|c|c|c|c|}
\hline \multirow{2}{*}{ Compound } & \multirow{2}{*}{$C \log p$} & \multirow{2}{*}{ Mol. Wt. } & \multicolumn{2}{|c|}{ BCG } & \multicolumn{2}{|c|}{ 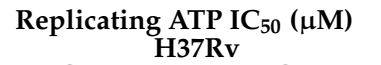 } & \multicolumn{2}{|c|}{ N0145 } \\
\hline & & & $-Q 203$ & +Q203 & -Q203 & +Q203 & $-Q 203$ & +Q203 \\
\hline 3 & 3.98 & 249.31 & $>25$ & $11.2 \pm 1.574$ & $>25$ & $27.2 \pm 4.613$ & $>25$ & $6.9 \pm 0.752$ \\
\hline $6 a$ & 4.86 & 317.31 & $>25$ & $5.1 \pm 0.501$ & $>25$ & $16.9 \pm 3.758$ & $>25$ & $2.6 \pm 0.581$ \\
\hline $7 \mathbf{a}$ & 5.01 & 333.31 & $>25$ & $2.1 \pm 0.205$ & $>25$ & $11.0 \pm 2.081$ & $>25$ & $1.1 \pm 0.719$ \\
\hline $8 a$ & 4.69 & 283.76 & $>25$ & $12.5 \pm 1.952$ & $>25$ & $29.7 \pm 5.336$ & $>25$ & $8.2 \pm 0.808$ \\
\hline $9 a$ & 5.21 & 375.36 & $>25$ & $3.9 \pm 0.490$ & $>25$ & $14.5 \pm 2.691$ & $>25$ & $2.3 \pm 0.853$ \\
\hline $10 a$ & 4.48 & 263.34 & $>25$ & $6.7 \pm 1.116$ & $>25$ & $20.7 \pm 3.624$ & $>25$ & $3.8 \pm 0.813$ \\
\hline $11 a$ & 3.9 & 279.34 & $>25$ & $13.7 \pm 2.107$ & $>25$ & $>25$ & $>25$ & $7.1 \pm 0.653$ \\
\hline $12 a$ & 5.81 & 305.42 & $>25$ & $0.8 \pm 0.144$ & $>25$ & $5.8 \pm 0.616$ & $>25$ & $0.1 \pm 0.017$ \\
\hline $13 a$ & 5.01 & 333.31 & $>25$ & $7.1 \pm 0.849$ & $>25$ & $22.5 \pm 3.912$ & $>25$ & $3.8 \pm 0.733$ \\
\hline $14 a$ & 4.43 & 293.36 & $>25$ & $5.8 \pm 0.867$ & $>25$ & $19.3 \pm 5.703$ & $>25$ & $3.2 \pm 0.204$ \\
\hline $15 a$ & 4.86 & 317.31 & $>25$ & $3.9 \pm 0.311$ & $>25$ & $17.0 \pm 2.945$ & $>25$ & $3.2 \pm 0.795$ \\
\hline 1 & 5.85 & 313.44 & $>25$ & $5.8 \pm 1.06$ & $>25$ & $18.9 \pm 9.03$ & $>25$ & $8.5 \pm 2.38$ \\
\hline 2 & 6.69 & 381.36 & $>25$ & $0.7 \pm 0.103$ & $>25$ & $6.3 \pm 0.349$ & $>25$ & $0.3 \pm 0.049$ \\
\hline BDQ & 7.25 & 555.52 & $0.17 \pm 0.019$ & $0.11 \pm 0.026$ & $0.06 \pm 0.019$ & $0.04 \pm 0.024$ & $0.05 \pm 0.003$ & $0.03 \pm 0.001$ \\
\hline Aurachin D & 6.79 & 363.55 & $>25$ & $2.9 \pm 0.317$ & $5.5 \pm 1.107$ & $7.3 \pm 1.973$ & $>25$ & $1.5 \pm 0.065$ \\
\hline
\end{tabular}

$\mathrm{IC}_{50}$ values were determined by ATP depletion in the presence and absence of Q203 (see Section 2.2 for details). IC 50 values were determined using GraphPad Prism 9. The values reflected in the table represent the average and standard deviation, which were calculated from the $\mathrm{IC}_{50}$ values of replicates from two experimental repeats. cLog $P$ was calculated by Perkin Elmer ChemDraw Professional 16.0. Bedaquiline (BDQ), ND-11992 (2) and aurachin D were used as positive control compounds.

Table 2. Screening of the second set of $N$-phenethylquinazolin-4-amines 16a-26a against cyt- $b d$ of $M$. bovis BCG and M. tuberculosis H37Rv by ATP readout along with known inhibitors ND-11992 (2), aurachin D and bedaquiline (BDQ) as positive controls.

\begin{tabular}{|c|c|c|c|c|c|c|c|c|}
\hline \multirow[t]{2}{*}{ Compound } & \multirow[t]{2}{*}{$C \log p$} & \multirow[t]{2}{*}{ Mol. Wt. } & \multicolumn{2}{|c|}{ BCG } & \multicolumn{2}{|c|}{$\underset{\text { R37Rv }}{\text { Replicating ATP IC }}$ IC $_{50}(\mu \mathrm{M})$} & \multicolumn{2}{|c|}{ N0145 } \\
\hline & & & $-Q 203$ & $+Q 203$ & $-Q 203$ & $+Q 203$ & $-Q 203$ & $+Q 203$ \\
\hline $16 a$ & 5.19 & 351.3 & $>25$ & $13.1 \pm 0.651$ & $>25$ & $28.8 \pm 5.054$ & $>25$ & $7.1 \pm 0.207$ \\
\hline $17 a$ & 5.51 & 347.33 & $>25$ & $7.5 \pm 0.580$ & $>25$ & $22.3 \pm 5.796$ & $>25$ & $3.4 \pm 0.559$ \\
\hline $18 a$ & 5.41 & 363.33 & $>25$ & $26.9 \pm 1.231$ & $>25$ & $>25$ & $>25$ & $17.1 \pm 0.220$ \\
\hline $19 a$ & 5.19 & 351.3 & $>25$ & $0.8 \pm 0.077$ & $>25$ & $7.6 \pm 1.079$ & $>25$ & $0.2 \pm 0.057$ \\
\hline $20 a$ & 5.76 & 367.75 & $>25$ & $>25$ & $>25$ & $>25$ & $>25$ & $>25$ \\
\hline $21 a$ & 5.91 & 412.21 & $>25$ & $6.7 \pm 0.591$ & $>25$ & $19.5 \pm 2.356$ & $>25$ & $4.9 \pm 0.226$ \\
\hline $22 a$ & 5.95 & 401.31 & $>25$ & $>25$ & $>25$ & $>25$ & $>25$ & $>25$ \\
\hline $23 a$ & 5.41 & 363.33 & $>25$ & $>25$ & $>25$ & $>25$ & $>25$ & $>25$ \\
\hline $24 a$ & 5.51 & 347.33 & $12.2 \pm 0.737$ & $3.1 \pm 0.369$ & $7.5 \pm 1.025$ & $11.7 \pm 1.252$ & $8.2 \pm 0.654$ & $2.9 \pm 0.140$ \\
\hline $25 a$ & 5.95 & 373.37 & $8.5 \pm 1.529$ & $4.0 \pm 0.604$ & $7.6 \pm 1.026$ & $11.7 \pm 1.036$ & $7.3 \pm 1.052$ & $2.1 \pm 0.347$ \\
\hline $26 a$ & 5.69 & 365.32 & $27.6 \pm 9.513$ & $6.5 \pm 0.751$ & $23.6 \pm 7.807$ & $16.9 \pm 3.822$ & $32.7 \pm 2.700$ & $4.6 \pm 0.219$ \\
\hline 2 & 6.69 & 381.36 & $>25$ & $0.7 \pm 0.103$ & $>25$ & $6.3 \pm 0.349$ & $>25$ & $0.3 \pm 0.049$ \\
\hline BDQQ & 7.25 & 555.52 & $0.17 \pm 0.019$ & $0.11 \pm 0.026$ & $0.06 \pm 0.019$ & $0.04 \pm 0.024$ & $0.05 \pm 0.003$ & $0.03 \pm 0.001$ \\
\hline Aurachin D & 6.79 & 363.55 & $>25$ & $2.9 \pm 0.317$ & $5.5 \pm 1.107$ & $7.3 \pm 1.973$ & $>25$ & $1.5 \pm 0.065$ \\
\hline
\end{tabular}

$\mathrm{IC}_{50}$ values were determined by ATP depletion in the presence and absence of Q203 (see 2.2 for details). $\mathrm{IC}_{50}$ values were determined using GraphPad Prism 9. The values given in the table represent the average and standard deviation, which were calculated from the $\mathrm{IC}_{50}$ values of replicates from two experimental repeats. $c \log P$ was calculated by Perkin Elmer ChemDraw Professional 16.0. Bedaquiline (BDQ), ND-11992 (2) and aurachin D were used as positive control compounds. 
3.1. Chemical Syntheses of the First Set of N-Phenethylquinazolin-4-Amines $(\mathbf{6} \boldsymbol{a}-\mathbf{1 5 a})$ and the Second Set of N-Phenethylquinazolin-4-Amines (16a-26a)

The first set of compounds (6a-15a) were prepared by reaction of 4-chlorquinazoline (5) with ten commercially available amines (6-15) using $S_{N}$ Ar conditions (basic or acidic, Scheme 1). These compounds were evaluated for their activity against cyt-bd in $M$. bovis BCG using an ATP readout (Table 1).

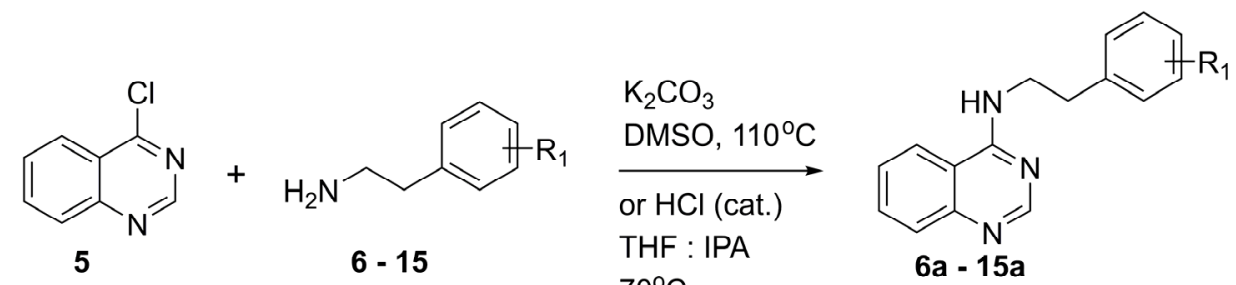<smiles>FC(F)(F)Oc1ccc(CCNc2ncnc3ccccc23)cc1</smiles><smiles>COc1ccc(CCNc2ncnc3ccccc23)cc1</smiles>
9 a $10 a$<smiles>CCOc1cccc(CCNc2ncnc3ccccc23)c1</smiles><smiles>FC(F)(F)c1ccccc1CCNc1ncnc2ccccc12</smiles>

Scheme 1. $\mathrm{S}_{N} A$ r conditions to prepare the first set of $N$-phenethylquinazolin-4-amines (6a-15a) for screening against cyt-bd of M. bovis BCG and M. tuberculosis H37Rv.

The second set of compounds (16a-26a) was prepared by reacting eight functionalized 4-chloroquinazolines (16-26) with 4-(trifluoromethoxy)benzylamine (7) using standard $\mathrm{S}_{\mathrm{N}} \mathrm{Ar}$ conditions (Scheme 2). As with the first set, the activity was determined against cyt-bd in M. bovis BCG and M. tuberculosis H37Rv by ATP readout (Table 2). 


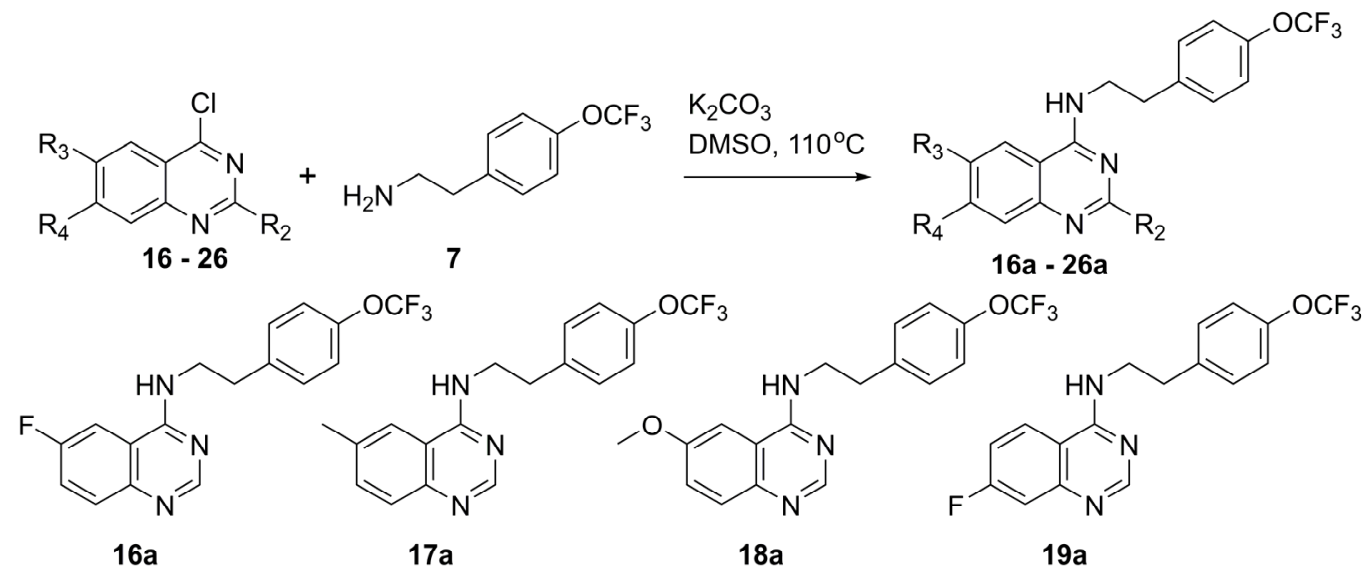<smiles>CCCOc1ccc(CCNc2ncnc3cc(Cl)ccc23)cc1CCNc1ncnc2cc(Br)ccc12</smiles><smiles>COc1ccc(CCNc2nc(C)nc3ccccc23)cc1</smiles><smiles>COc1ccc(CCNc2nc(C3CC3)nc3ccccc23)cc1</smiles><smiles>Cc1nc(NCCc2ccc(OC(F)(F)F)cc2)c2ccc(F)cc2n1</smiles>

Scheme 2. $\mathrm{S}_{N} A r$ conditions to prepare the second set of $N$-phenethylquinazolin-4-amines (16a-26a) for screening against cyt-bd of M. bovis BCG and M. tuberculosis H37Rv.

\subsection{Structure-Activity Relationship Studies}

The structure-activity-relationship (SAR) studies revealed that the cyt-bd of $M$. bovis BCG was more sensitive to inhibition than M. tuberculosis H37Rv (Table 1). This mirrors the trend observed previously and is indicative that there is a greater expression of cyt- $b d$ in the lab-adapted M. tuberculosis H37Rv strain relative to the M. bovis BCG or "fresh" Mtb clinical isolate N0145 [13]. This is further supported by the activity of aurachin D [7], a known synergistic cyt- $b d$ inhibitor of Mtb, which had greater activity against $M$. bovis BCG in our ATP assay relative to $M$. tuberculosis $\mathrm{H} 37 \mathrm{Rv}(2.9 \mu \mathrm{M}$ vs. $5.5 \mu \mathrm{M}$, respectively; Table 1). In addition, based on our assay, compounds $\mathbf{6 a - 1 5 a}$ are all specific inhibitors of cyt- $b d$ as they show no activity against wild-type (WT) M. bovis BCG or M. tuberculosis H37Rv (Mtb$\mathrm{H} 37 \mathrm{Rv}$ ), whereas the positive control bedaquiline (BDQ), a selective ATP synthase inhibitor, remains very potent against both strains (Table 1 ). Interestingly, aurachin D showed the expected synergy with Q203 against mycobacterial strains but was unexpectedly active against wild-type Mtb-H37Rv (Table 1).

SAR trends revealed that the pendant aryl group can accommodate a variety of steric and electronic changes and retain good activity (Table 1). Evaluation of the compounds with electron withdrawing substituents $\mathbf{6 a}\left(p-\mathrm{CF}_{3}\right), 7 \mathbf{a},\left(p-\mathrm{OCF}_{3}\right), 8 \mathbf{a}(p-\mathrm{Cl}), \mathbf{9 a}\left(p-\mathrm{SF}_{5}\right)$, indicated that $7 \mathrm{a}$ is the most active compound with $\mathrm{IC}_{50}$ values of $2.1 \mu \mathrm{M}$ against $M$. bovis BCG and $11 \mu \mathrm{M}$ against Mtb H37Rv. We explored electron withdrawing groups at the metaand ortho-positions of the pendant aryl group by comparing compound sets $7 \mathbf{a}\left(\right.$ para- $\left.-\mathrm{OCF}_{3}\right)$ and 13a $\left(\right.$ meta-OCF 3 ) and $\mathbf{6 a}\left(\right.$ para $\left.-\mathrm{CF}_{3}\right)$ and 15a $\left(\right.$ ortho- $\left.-\mathrm{CF}_{3}\right)$. We found that the meta-OCF 3 compound (13a) position was less active compared to the para- $\mathrm{OCF}_{3}(\mathbf{1 7 a})$. Whereas incorporation of substituents in the ortho-position gave slightly improved potency against 
M. bovis but equal activity (within error) in Mtb-H37Rv as demonstrated by comparing 6a and 15a. When evaluating the compounds with electron donating substituents, 10a $\left(p-\mathrm{CH}_{3}\right), \mathbf{1 1 a}\left(p-\mathrm{OCH}_{3}\right)$, and 12a (p-tert-butyl), 12a was found to be significantly more active with $\mathrm{IC}_{50}$ values of $0.8 \mu \mathrm{M}$ against $M$. bovis BCG and $5.8 \mu \mathrm{M}$ against Mtb H37Rv. That is an improvement over thieno[3,2-d]pyrimidine (1) and activity on par with the optimized quinazoline ND-11992 (2). This is particularly interesting as it follows the Topliss aromatic substitution decision tree pattern [23] where one would first evaluate the phenyl (3), then para-chloro (8a) and if equally potent (as it is in our assays) then prepare the tert-butyl (12a) to derive the most potent compound. While the Topliss approach can often lead to compounds with improved activity it also directs the syntheses of compounds which often have metabolic liabilities $\left(\mathrm{CH}_{3}, \mathrm{OCH}_{3}, t\right.$-butyl) or toxic pharmacophores $\left(\mathrm{NH}_{2}, \mathrm{NO}_{2}, \mathrm{I}\right)$. For this reason, we chose $\mathbf{7 a}$ which bears a para-trifluoromethoxy group (which is prevalent in various anti-TB drugs such as delamanid, pretomanid, and telacebec) as the basis for our second set of compounds (Table 2) because it possesses good activity ( $\mathrm{IC}_{50}{ }^{\prime} \mathrm{s}$ of 2.1 and $11 \mu \mathrm{M}$ against BCG and Mtb-H37Rv, respectively).

The SAR trends for alteration of the quinazoline core suggest an interplay between both steric and electronic effects (Table 2). For instance, the 6-position was probed with three substituents-fluoro (16a), methyl (17a) and methoxy (18a) - which resulted in a wide spectrum of activity. The 6-methyl analog (17a) was the most active against $M$. bovis BCG $\left(\mathrm{IC}_{50}\right.$ of $\left.7.5 \mu \mathrm{M}\right)$ but weakly active against Mtb H37Rv ( $\mathrm{IC}_{50}$ of $\left.22 \mu \mathrm{M}\right)$. Whereas the fluoro (16a) and methoxy (18a) analogs had diminished activity against $M$. bovis BCG ( $\mathrm{IC}_{50}$ of $13 \mu \mathrm{M}$ and $27 \mu \mathrm{M}$, respectively) and both were $>25 \mu \mathrm{M}$ against Mtb H37Rv. The 7-position was explored with five different substituents-fluoro (19a), chloro (20a), bromo (21a), trifluoromethoxy (22a) and methoxy (23a) - resulting in only two active compounds (19a and 21a). The 7-fluoro analog (19a) displayed good activity against both mycobacterial strains ( $\mathrm{IC}_{50}$ of $0.8 \mu \mathrm{M}$ and $7.6 \mu \mathrm{M}$; respectively) and the 7-bromo (21a) showed much better activity against $M$. bovis BCG than Mtb H37Rv ( $\mathrm{IC}_{50}$ of $7 \mu \mathrm{M}$ and $20 \mu \mathrm{M}$; respectively). The most interesting SAR was observed with substitution of the quinazoline 2-position with methyl (24a) and cyclopropyl (25a) groups. Both compounds displayed good activity range

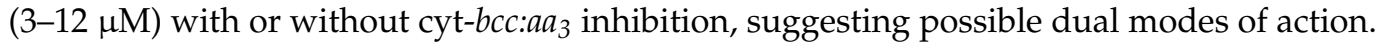
Lastly, one compound (26a) bore both 7-fluoro and 2-methyl quinazoline substituents which biased activity back towards cyt- $b d\left(\mathrm{IC}_{50}\right.$ of 7 and $17 \mu \mathrm{M}$ with Q203, and $28 \mu \mathrm{M}$ and $24 \mu \mathrm{M}$ without Q203; respectively)

Our previous work had shown that the laboratory adapted Mtb H37Rv strain over expresses cyt- $b d$ resulting in higher $\mathrm{IC}_{50}$ values than those observed using a clinical $\mathrm{Mtb}$ isolate [24]. These higher $\mathrm{IC}_{50}$ values render compound ranking more challenging. To gain greater insight on the activity of these compounds, they were re-screened against the clinical Mtb isolate N0145 strain. As observed previously with the thieno[3,2- $d]$ pyrimidin-4amine class of cyt- $b d$ oxidase inhibitors [13], these compounds displayed greatly improved activity against the Mtb clinical isolate likely due to lower expression of cyt-bd within clinical isolates as compared to lab-adapted strains like H37Rv [12]. Two compounds, 7a and 12a, from the first set were more active than or comparable in activity to aurachin $D$ (0.1 and $1.1 \mu \mathrm{M}$ vs. $1.5 \mu \mathrm{M}$, respectively). While six additional compounds (6a, 9a, 10a, 13a-15a) had IC 50 values below $5 \mu \mathrm{M}$ against N0145-Mtb. In the second set of compounds, only 19a had activity superior to aurachin $\mathrm{D}(0.2 \mu \mathrm{M}$ vs. $1.5 \mu \mathrm{M}$, respectively) and four additional compounds (17a, 21a, 24a-26a) had activity below $5 \mu \mathrm{M}$. The two most potent compounds, 12a and 19a, had slightly improved activity relative to ND-11992 which was active in the murine infection model of tuberculosis. However, preliminary metabolic stability assessment of these compounds against human and rat microsomes indicate much more rapid metabolism than that of ND-11992 (data not shown).

\section{Conclusions}

The described $\mathrm{N}$-phenethylquinazolin-4-amine class of compounds are mycobacterial cyt- $b d$ inhibitors. Through focused SAR, the activity of the hit compound $3\left(\mathrm{IC}_{50}=6.9 \mu \mathrm{M}\right.$ 
against Mtb N0145) was improved with 14 of the 22 analogs (64\%) and two, 12a and 19a, had sub-micromolar activity (0.1 and $0.2 \mu \mathrm{M}$ against Mtb N0145, respectively). Future work includes identification of more efficacious compounds through additional SAR around the quinazoline core. These results will be reported in due course.

\section{Patents}

US Provisional Application No. 62/783,984.

Supplementary Materials: The following are available online at https://www.mdpi.com/article/10 .3390 /app11199092/s1, Figures S1-S60: ${ }^{1} \mathrm{H}$ NMR, ${ }^{13} \mathrm{C}$ NMR and ${ }^{19} \mathrm{~F}$ NMR Spectra of all compounds; Figure S61: ATP dose response curves of Q203 in M. bovis BCG, M. tuberculosis H37Rv, and M. tuberculosis N0145.

Author Contributions: G.C.M. designed the compounds, which were prepared and characterized by S.M.H. and G.C.M.; K.P. designed all the biological assays which were carried out by B.S.L. and N.P.K.; M.J.M. provided data interpretation and scientific direction. All authors assisted in the drafting and editing of the manuscript. All authors have read and agreed to the published version of the manuscript.

Funding: M.J.M. and G.C.M. acknowledge funding from the NIH R37AI054193. K.P. and G.C.M. acknowledge funding from the NIH R01AI139465. This study was partially funded by the National Research Foundation (NRF) Singapore under its NRF Competitive Research Programme (Project Award Number NRF-CRP18-2017-01, to K.P.) and its NRF Investigatorship programme (Award Number NRF-I06-2020-0012, to K.P.). B.S.L. is supported by a Nanyang President's Graduate Scholarship from NTU.

Acknowledgments: G.C.M. acknowledges Jennifer DuBois for persistent scientific discussions, Mary Cloninger for mentoring guidance and Kate Marshall for NMR assistance.

Conflicts of Interest: Hsiri Therapeutics has licensed this technology. M.J.M. and G.C.M. own equity in Hsiri. M.J.M. is CSO of Hsiri. G.C.M. and K.P. are consultants to Hsiri. Hsiri Therapeutics did not fund this study and was not involved in study design or interpretation.

\section{References}

1. World Health Organization. Global Tuberculosis Report 2020; World Health Organization: Geneva, Switzerland, 2020.

2. Iacobino, A.; Fattorini, L.; Giannoni, F. Drug-Resistant Tuberculosis 2020: Where We Stand. Appl. Sci. 2020, 10, 2153. [CrossRef]

3. Hasenoehrl, E.J.; Wiggins, T.J.; Berney, M. Bioenergetic Inhibitors: Antibiotic Efficacy and Mechanisms of Action in Mycobacterium tuberculosis. Front. Cell. Infect. Microbiol. 2021, 10, 26. [CrossRef] [PubMed]

4. Aung, H.L.; Berney, M.; Cook, G.M. Hypoxia-Activated Cytochrome bd Expression in Mycobacterium smegmatis Is Cyclic AMP Receptor Protein Dependent. J. Bacteriol. 2014, 196, 3091-3097. [CrossRef] [PubMed]

5. Bald, D.; Villellas, C.; Lu, P.; Koul, A. Targeting Energy Metabolism in Mycobacterium tuberculosis, a New Paradigm in Antimycobacterial Drug Discovery. Mbio 2017, 8, 11. [CrossRef] [PubMed]

6. Kalia, N.P.; Hasenoehrl, E.J.; Ab Rahman, N.B.; Koh, V.H.; Ang, M.L.T.; Sajorda, D.R.; Hards, K.; Gruber, G.; Alonso, S.; Cook, G.M.; et al. Exploiting the synthetic lethality between terminal respiratory oxidases to kill Mycobacterium tuberculosis and clear host infection. Proc. Natl. Acad. Sci. USA 2017, 114, 7426-7431. [CrossRef] [PubMed]

7. Lu, P.; Asseri, A.H.; Kremer, M.; Maaskant, J.; Ummels, R.; Lill, H.; Bald, D. The anti-mycobacterial activity of the cytochrome bcc inhibitor Q203 can be enhanced by small-molecule inhibition of cytochrome bd. Sci. Rep. 2018, 8, 7. [CrossRef] [PubMed]

8. Beites, T.; O’Brien, K.; Tiwari, D.; Engelhart, C.A.; Walters, S.; Andrews, J.; Yang, H.J.; Sutphen, M.L.; Weiner, D.M.; Dayao, E.K.; et al. Plasticity of the Mycobacterium tuberculosis respiratory chain and its impact on tuberculosis drug development. Nat. Commun. 2019, 10, 12. [CrossRef] [PubMed]

9. Cook, G.M.; Hards, K.; Vilcheze, C.; Hartman, T.; Berney, M. Energetics of Respiration and Oxidative Phosphorylation in Mycobacteria. Microbiol. Spectr. 2014, 2, 20. [CrossRef] [PubMed]

10. Pethe, K.; Bifani, P.; Jang, J.C.; Kang, S.; Park, S.; Ahn, S.; Jiricek, J.; Jung, J.Y.; Jeon, H.K.; Cechetto, J.; et al. Discovery of Q203, a potent clinical candidate for the treatment of tuberculosis. Nat. Med. 2013, 19, 1157-1160. [CrossRef] [PubMed]

11. Matsoso, L.G.; Kana, B.D.; Crellin, P.K.; Lea-Smith, D.J.; Pelosi, A.; Powell, D.; Dawes, S.S.; Rubin, H.; Coppel, R.L.; Mizrahi, V. Function of the cytochrome bc(1)-aa(3) branch of the respiratory network in mycobacteria and network adaptation occurring in response to its disruption. J. Bacteriol. 2005, 187, 6300-6308. [CrossRef] [PubMed]

12. Lee, B.S.; Hards, K.; Engelhart, C.A.; Hasenoehrl, E.J.; Kalia, N.P.; Mackenzie, J.S.; Sviriaeva, E.; Chong, S.M.S.; Manimekalai, M.S.S.; Koh, V.H.; et al. Dual inhibition of the terminal oxidases eradicates antibiotic-tolerant Mycobacterium tuberculosis. Embo Mol. Med. 2021, 13, 16. [CrossRef] [PubMed] 
13. Hopfner, S.M.; Lee, B.S.; Kalia, N.P.; Miller, M.J.; Pethe, K.; Moraski, G.C. Structure guided generation of thieno 3,2-d pyrimidin-4amine Mycobacterium tuberculosis bd oxidase inhibitors. RSC Med. Chem. 2021, 12, 73-77. [CrossRef] [PubMed]

14. Mohamed, T.; Rao, P.P.N. 2,4-Disubstituted quinazolines as amyloid-beta aggregation inhibitors with dual cholinesterase inhibition and antioxidant properties: Development and structure-activity relationship (SAR) studies. Eur. J. Med. Chem. 2017, 126, 823-843. [CrossRef] [PubMed]

15. Rewcastle, G.W.; Denny, W.A.; Bridges, A.J.; Zhou, H.R.; Cody, D.R.; McMichael, A.; Fry, D.W. Tyrosine Kinase Inhibitors. 5. Synthesis and Structure-Activity Relationships for 4-[(Phenylmethyl)amino]- and 4-(Phenylamino)quinazolines as Potent Adenosine 5'-Triphosphate Binding Site Inhibitors of the Tyrosine Kinase Domain of the Epidermal Growth Factor Receptor. J. Med. Chem. 1995, 38, 3482-3487. [CrossRef] [PubMed]

16. Lee, Y.B.; Ahn, C.H. Quinazoline Derivatives and Therapeutic Use Therof. U.S. Patent No. 8,404,698, 26 March 2013.

17. Dreikorn, B.A.; Jourdan, G.P.; Suhr, R.G. Quinazoline Derivatives. U.S. Patent 5,411,963, 2 May 1995.

18. Tobe, M.; Isobe, Y.; Tomizawa, H.; Matsumoto, M.; Obara, F.; Nagasaki, T.; Hayashi, H. Structure-activity relationships of quinazoline derivatives: Dual-acting compounds with inhibitory activities toward both TNF-alpha production and T cell proliferation. Bioorganic Med. Chem. Lett. 2001, 11, 545-548. [CrossRef]

19. Tobe, M.; Isobe, Y.; Tomizawa, H.; Nagasaki, T.; Takahashi, H.; Fukazawa, T.; Hayashi, H. Discovery of quinazolines as a novel structural class of potent inhibitors of NF-kappa B activation. Bioorganic Med. Chem. 2003, 11, 383-391. [CrossRef]

20. Tsai, K.C.; Teng, L.W.; Shao, Y.M.; Chen, Y.C.; Lee, Y.C.; Li, M.Y.; Hsiao, N.W. The first pharmacophore model for potent NF-kappa B inhibitors. Bioorganic Med. Chem. Lett. 2009, 19, 5665-5669. [CrossRef] [PubMed]

21. Matsumoto, J.; Sakamoto, K.; Shinjyo, N.; Kido, Y.; Yamamoto, N.; Yagi, K.; Miyoshi, H.; Nonaka, N.; Katakura, K.; Kita, K.; et al. Anaerobic NADH-fumarate reductase system is predominant in the respiratory chain of Echinococcus multilocularis, providing a novel target for the chemotherapy of alveolar echinococcosis. Antimicrob. Agents Chemother. 2008, 52, 164-170. [CrossRef] [PubMed]

22. Yuan, J.; Ma, D.; Liu, J.; Zhang, L. Potent Small Molecule Inhibitors of Autophagy, and Methods of Use Thereof. U.S. Patent Application No. 13/382,572, 21 July 2009.

23. Topliss, J.G. Utilization of Operational Schemes for Analog Synthesis in Drug Design. J. Med. Chem. 1972, $15,1006-1011$. [CrossRef] [PubMed]

24. Arora, K.; Ochoa-Montano, B.; Tsang, P.S.; Blundell, T.L.; Dawes, S.S.; Mizrahi, V.; Bayliss, T.; Mackenzie, C.J.; Cleghorn, L.A.T.; Ray, P.C.; et al. Respiratory Flexibility in Response to Inhibition of Cytochrome c Oxidase in Mycobacterium tuberculosis. Antimicrob. Agents Chemother. 2014, 58, 6962-6965. [CrossRef] [PubMed] 